\title{
Exocyclic Enamines of Pyrrolo[1,2-a]quinoxalines Generated by 1,3-dipolar Cycloaddition Reactions of Benzimidazolium Ylides to Activated Alkynes
}

\author{
ALINA NICOLESCU ${ }^{1,2}$, EMILIAN GEORGESCU ${ }^{3}$, FLOREA DUMITRASCU ${ }^{1}$, \\ FLORENTINA GEORGESCU ${ }^{4}$, FLORINA TEODORESCU ${ }^{1}$, CONSTANTIN DRAGHICI ${ }^{2}$, \\ MINO R. CAIRA ${ }^{5, *}$, CALIN DELEANU ${ }^{1,2 *}$ \\ ${ }^{1}$ C. D. Nenitescu Centre of Organic Chemistry, Romanian Academy, 202B Independentei, 060023, Bucharest, Romania \\ ${ }^{2}$ Petru Poni Institute of Macromolecular Chemistry, Romanian Academy, 41A Grigore Ghica Voda, 700487, Iasi, Romania \\ ${ }^{3}$ Chimcomplex Research Center, 1 Uzinei Str., 240050, Ramnicu Valcea, Romania \\ ${ }^{4}$ Enpro Soctech Com SRL, 31 Sfantul Elefterie Str., 050524, Bucharest, Romania \\ ${ }^{5}$ Department of Chemistry, University of Cape Town, Rondebosch 7701, South Africa
}

\begin{abstract}
The reactions of $N$-acetonylbenzimidazolium chlorides with various activated alkynes, in the presence of bases, led under mild conditions to a mixture of pyrrolo[1,2-a]benzimidazoles (3), 4methylene-pyrrolo[1,2-a]quinoxaline derivatives (4) and pyrrolo[1,2-a]quinoxalin-4-one derivatives (5). The exocyclic enamine derivatives 4 have been fully characterized by multinuclear NMR spectroscopy and X-ray crystallography. A mechanism rationalizing the formation of the enamine derivatives is proposed.
\end{abstract}

Keywords: Nitrogen heterocycles, Pyrrolo[1,2-a]quinoxaline, Pyrrolo[1,2-a]benzimidazole, Pyrrolo[1,2-a]quinoxalinone, Enamines, Ylides, Cycloaddition

\section{Introduction}

Pyrrolo[1,2-a]benzimidazole and pyrrolo[1,2-a]quinoxaline cores are extensively investigated mainly due to their presence in numerous bioactive compounds. Thus, pyrrolo[1,2-a]benzimidazole scaffold is present in a range of DNA cross-linkers mimicking the mitomycin antitumor activity against a range of human cell lines [1-4], and many pyrrolo[1,2-a]benzimidazole derivatives showed beneficial properties on central nervous system disorders [5]. Pyrrolo[1,2-a]quinoxaline skeleton is present in numerous compounds that proved potent inhibitors of the human protein kinase CK2 [6]. Many pyrrolo[1,2-a] quinoxaline derivatives showed anti-tuberculosis [7], antiparasitic [8], antiallergic [9], and central dopamine antagonist activities [10]. Several pyrrolo[1,2-a]quinoxaline derivatives showed effectiveness in the treatment of cancer and disorders associated with angiogenesis function [11], and proved highly selective agonist affinity for the serotonin receptors [12].

Synthetic routes towards pyrrolo[1,2-a]benzimidazole and pyrrolo[1,2-a]quinoxaline derivatives, as well as their chemical and biological properties, have been reviewed [13-15]. Many pyrrolo[1,2$a]$ benzimidazole and pyrrolo[1,2-a]quinoxaline compounds have been synthesized based on the classical 1,3-dipolar cycloaddition reaction of benzimidazolium ylides, generated in situ from the corresponding benzimidazolium salts in the presence of organic or inorganic bases, with electron-deficient alkynes or alkenes[16-22]. Other synthetic approaches have been recently employed in a growing interest for these compounds [23-25].

Our group investigated the reactivity of benzimidazolium ylides towards various dipolarophiles in 1,3-dipolar cycloadditions and developed novel approaches towards pyrrolo[1,2-a]benzimidazole and pyrrolo[1,2-a]quinoxaline compounds [26-30]. We found that depending on the reaction conditions, either pyrrolo[1,2-a]benzimidazole derivatives or pyrrolo[1,2-a]quinoxaline derivatives can be obtained

$\overline{\text { *email: calin.deleanu@yahoo.com; Mino.Caira@uct.ac.za }}$ 
as major products in these cycloadditions[26-30]. Moreover, the structures of obtained pyrrolo[1,2a]quinoxalines depend on the structures of the ylide carbon substituents. For instance, pyrrolo[1,2a]quinoxalin-4-ones are always the major reaction products when 1-benzyl-3-alkoxycarbonylmethylbenzimidazolium ylides are intermediate 1,3-dipoles [20, 27]. 4-Phenyl-pyrrolo[1,2a]quinoxaline derivatives are the major reaction products when starting from 1-alkyl-3phenacylbenzimidazolium bromides and activated alkynes in the presence of epoxides, while in the presence of tertiary amines, 4-hydroxy-4,5-dihydropyrrolo[1,2-a]quinoxaline derivatives and pyrrolo[1,2-a]quinoxalinium quaternary salts are the major reaction products [29]. In the same reactions, pyrrolo[1,2-a]benzimidazole derivatives are obtained in good yields when tertiary amines and an oxidant are used together $[29,30]$.

The continuing interest in pyrrolo[1,2- $a]$ benzimidazole and pyrrolo[1,2- $a$ ]quinoxaline chemistry prompted us to investigate 1,3-dipolar cycloaddition reactions of benzimidazolium ylides, generated from the corresponding $N$-acetonyl-benzimidazolium salts, with various acetylenic dipolarophiles.

\section{Materials and methods \\ General}

Melting points were measured on a Boëtius hot plate microscope and are uncorrected. IR spectra were recorded on a Nicolet Impact 410 spectrometer, in $\mathrm{KBr}$ pellets. The high performance liquid chromatography (HPLC) analyses were performed with an Agilent Chromatograph 1200 Series at room temperature by isocratic elution of acetonitrile on an Agilent Zorbax SB-C18 (250x4.6) column with flow rate $1.0 \mathrm{~mL} / \mathrm{min}$.

Most of the NMR spectra have been recorded on a Bruker Avance III 400 instrument operating at 400.1, 100.6 and 40.6 MHz for ${ }^{1} \mathrm{H},{ }^{13} \mathrm{C}$, and ${ }^{15} \mathrm{~N}$ nuclei respectively. Samples were transferred in $5 \mathrm{~mm}$ Wilmad $507 \mathrm{NMR}$ tubes and recorded with either a $5 \mathrm{~mm}$ multinuclear inverse detection $\mathrm{z}$-gradient probe $\left({ }^{1} \mathrm{H}\right.$ spectra and all $\mathrm{H}-\mathrm{C} / \mathrm{H}-\mathrm{N} 2 \mathrm{D}$ experiments) or with a $5 \mathrm{~mm}$ four nuclei direct detection $\mathrm{z}$ gradient probe $\left({ }^{1} \mathrm{H}\right.$ and ${ }^{13} \mathrm{C}$ spectra). Chemical shifts are reported in $\delta$ units (ppm) and were referenced to internal TMS for ${ }^{1} \mathrm{H}$ chemical shifts, to the internal deuterated solvent for ${ }^{13} \mathrm{C}$ chemical shifts $\left(\mathrm{CDCl}_{3}\right.$ referenced at $77.0 \mathrm{ppm}$ and DMSO referenced at $39.4 \mathrm{ppm})$, and referenced to liquid ammonia (0.0 ppm) using nitromethane (380.2 ppm) as external standard for ${ }^{15} \mathrm{~N}$ chemical shifts. Unambiguous 1D NMR signal assignments were made based on 2D NMR homo- and heterocorrelation. H,H-COSY, H,CHSQC, H,C-HMBC and H,H-ROESY experiments were recorded using standard pulse sequences in the version with $\mathrm{z}$-gradients, as delivered by Bruker with TopSpin 2.1 PL6 spectrometer control and processing software. The individual chemical shifts, multiplicities and coupling constants for overlapping signals have been obtained from undecoupled H,C-HSQC experiments recorded using the pulse sequence described by S. Simova [31]. The ${ }^{15} \mathrm{~N}$ chemical shifts were obtained as projections from the 2D indirectly detected H,N-HMBC spectra, employing a standard pulse sequence in the version with z-gradients as delivered by Bruker (TopSpin 2.1 PL6). Some reaction controls have been performed using a Varian Gemini $300 \mathrm{NMR}$ spectrometer operating at 300 and $75 \mathrm{MHz}$ for ${ }^{1} \mathrm{H}$ and ${ }^{13} \mathrm{C}$ nuclei respectively.

X-ray crystallography for structure $\mathbf{4 e}$ was performed by direct methods from intensity data measured on a Nonius KappaCCD four-circle diffractometer with the crystal cooled to 173(2) K. Crystallographic data for 4e have been deposited at the Cambridge Crystallographic Data Centre (CCDC 1527017). Copies of the data can be obtained free of charge on application to the CCDC, 12 Union Road, Cambridge CB2 IEZ, UK. Fax: +44-(0)1223-336033 or e-mail: deposit@ccdc.cam.ac.uk.

Elemental analyses for $\mathrm{C}, \mathrm{H}$ and $\mathrm{N}$ were obtained using a COSTECH Instruments EAS32. Satisfactory microanalyses for all new compounds were obtained.

Benzimidazole, 1-methylbenzimidazole, ethyl propiolate, dimethyl acetylenedicarboxylate, 3-butyn2-one, chloroacetone and triethylamine were purchased from Aldrich and used without further purification. 1-Benzylbenzimidazole, respectively 1-p-methylbenzylbenzimidazole, was obtained from 
benzimidazole and benzyl chloride, respectively $p$-methylbenzyl chloride. $N$-acetonylbenzimidazolium chlorides (1) were obtained from 1-substituted benzimidazole and chloroacetone (2) in acetone, according to previously reported methods [32]. Tetrapyridinecobalt(II)dichromate (TPCD) was obtained according to the previously reported method [33]. Chloroform used was stored on anhydrous $\mathrm{K}_{2} \mathrm{CO}_{3}$.

\section{General procedure for the reaction of $\mathrm{N}$-acetonylbenzimidazolium chlorides (1) with activated alkynes (2) in the presence of triethyl amine in acetonitrile}

To a mixture of 3-acetonylbenzimidazolium chloride $\mathbf{1}(3 \mathrm{mmol})$ in $25 \mathrm{~mL}$ of acetonitrile, an alkyne dipolarophile $2(3.3 \mathrm{mmol})$ and $0.63 \mathrm{~mL}(4.5 \mathrm{mmol})$ of triethylamine were added under stirring. The reaction mixture was heated at reflux for $3 \mathrm{~h}$, cooled and poured under stirring into $60 \mathrm{~mL}$ of water and extracted with $\mathrm{CHCl}_{3}(2 \times 60 \mathrm{~mL})$. The combined extracts were dried on $\mathrm{Na}_{2} \mathrm{SO}_{4}$ anh., the solvent was partly removed in vacuo and the residue chromatographed on $\mathrm{SiO}_{2}$ packed column by eluting with EtOAc:hexane $(1: 4 \mathrm{v} / \mathrm{v})$.

General procedure for the reaction of $\mathrm{N}$-acetonylbenzimidazolium chlorides (1) with activated alkynes (2) in the presence of triethyl amine and TPCD in DMF

To a mixture of 3-acetonylbenzimidazolium chloride 1 (3 mmol) in $25 \mathrm{~mL}$ of DMF, an alkyne dipolarophile $2(3.3 \mathrm{mmol}) 0.75 \mathrm{~g}(1.22 \mathrm{mmol})$ TPCD and $0.63 \mathrm{~mL}(4.5 \mathrm{mmol})$ triethylamine were added. The reaction mixture was heated at $90{ }^{\circ} \mathrm{C}$ for $3 \mathrm{~h}$, then cooled and poured under stirring into $40 \mathrm{~mL}$ of $\mathrm{HCl}\left(5 \%\right.$ in water) and extracted with $\mathrm{CHCl}_{3}(2 \times 60 \mathrm{~mL})$. The combined extracts were dried on $\mathrm{Na}_{2} \mathrm{SO}_{4}$ anh., the solvent was partly removed in vacuo and the residue chromatographed on a $\mathrm{SiO}_{2}$ packed column by eluting with EtOAc:hexane (1:4 v/v).

\section{Ethyl 1-acetyl-4-methyl-4H-pyrrolo[1,2-a]benzimidazole-3-carboxylate (3a)}

Pale yellow crystals, mp 173-174 ${ }^{\circ} \mathrm{C}$. FT-IR $\left(\mathrm{KBr}, \mathrm{cm}^{-1}\right)$ : 2979, 1688, 1628, 1579, 1505, 1487, 1386, 1203, 1151, 1087, 1060. ${ }^{1} \mathrm{H} \mathrm{NMR}\left(\mathrm{CDCl}_{3}, 400 \mathrm{MHz}\right) \delta \mathrm{ppm}: 1.40\left(\mathrm{t}, 3 \mathrm{H}, J 7.1 \mathrm{~Hz}, \mathrm{CH}_{3}-\mathrm{Et}\right), 2.46(\mathrm{~s}, 3 \mathrm{H}$, $\mathrm{CH}_{3} \mathrm{CO}$ ), 4.12 (s, 3H, CH $\left.3-\mathrm{N}\right), 4.29$ (q, 2H, J 7.2 Hz, $\left.\mathrm{CH}_{2}-\mathrm{Et}\right), 7.19$ (d, 1H, J 8.0 Hz, H-5), 7.20 (t, 1H, $J 8.0 \mathrm{~Hz}, \mathrm{H}-7), 7.31$ (t, 1H, J 7.3 Hz, H-6), 7.54 (s, 1H, H-2), 8.85 (d, 1H, J 8.2 Hz, H-8). ${ }^{13} \mathrm{C}$ NMR $\left(\mathrm{CDCl}_{3}, 100 \mathrm{MHz}\right) \delta \mathrm{ppm}: 14.5\left(\mathrm{CH}_{3}-\mathrm{Et}\right), 25.7\left(\mathrm{CH}_{3} \mathrm{CO}\right), 31.6\left(\mathrm{CH}_{3}-\mathrm{N}\right), 59.9\left(\mathrm{CH}_{2}-\mathrm{Et}\right), 92.0(\mathrm{C}-3)$, 108.8 (CH-5), $117.0(\mathrm{CH}-8), 121.0(\mathrm{CH}-7), 121.6$ (C-1), $124.0(\mathrm{CH}-6), 126.8$ (C-8a), $127.0(\mathrm{CH}-2)$, 136.6 (C-4a), 143.3 (C-3a), 163.3 (COO), 184.7 (CO). ${ }^{15} \mathrm{~N} \mathrm{NMR}\left(\mathrm{CDCl}_{3}, 40 \mathrm{MHz}\right) \delta \mathrm{ppm:} 106.8(\mathrm{~N}-4)$, 172.2 (N-9). Anal. Calcd. for $\mathrm{C}_{16} \mathrm{H}_{16} \mathrm{~N}_{2} \mathrm{O}_{3}$ (284.31): C, 67.59; H, 5.67; N, 9.85\%. Found: C, 67.70; H, $5.72 ; \mathrm{N}, 9.80 \%$.

\section{1-Acetyl-4-benzoyl-4-methyl-4H-pyrrolo[1,2-a]benzimidazole (3b)}

Pale yellow crystals, mp 151-153 ${ }^{\circ} \mathrm{C}$. FT-IR $\left(\mathrm{KBr}, \mathrm{cm}^{-1}\right): 2961,2920,1648,1619,1558,1506,1450$, 1340, 1260, 1160, 1018. ${ }^{1} \mathrm{H}$ NMR (CDCl3, $\left.400 \mathrm{MHz}\right) \delta \mathrm{ppm}: 2.47$ (s, 3H, $\left.\mathrm{CH}_{3} \mathrm{CO}\right), 4.32\left(\mathrm{~s}, 3 \mathrm{H}, \mathrm{CH}_{3}-\right.$ N), 7.35 (t, 1H, J 7.1 Hz, H-7), $7.41(\mathrm{~d}, 1 \mathrm{H}, J 7.3 \mathrm{~Hz}, \mathrm{H}-5), 7.43$ (s, 1H, H-2), 7.46 (t, 1H, J 7.3 Hz, H6), 7.53 (t, 2H, J 7.6 Hz, H-3"), 7.60 (t, 1H, J 7.3 Hz, H-4”), 7.87 (d, 2H, J 8.3 Hz, H-2"), 9.00 (d, 1H, $J 8.3 \mathrm{~Hz}, \mathrm{H}-8) .{ }^{13} \mathrm{C} \mathrm{NMR}(\mathrm{CDCl} 3,100 \mathrm{MHz}) \delta \mathrm{ppm}: 25.8\left(\mathrm{CH}_{3} \mathrm{CO}\right), 32.4\left(\mathrm{CH}_{3}-\mathrm{N}\right), 101.9(\mathrm{C}-3), 109.4$ (CH-5), 117.5 (CH-8), 121.8 (CH-7), 122.1 (C-1), 124.6 (CH-6), 127.0 (C-8a), 128.4 (CH-3”), 128.9 (CH-2), 129.1 (CH-2”), 131.5 (CH-4”), 137.0 (C-4a), 140.1 (C-1”), 144.8 (C-3a), 185.2 (COCH (C), $188.7^{(\mathrm{C}}$ $(\mathrm{COPh}) .{ }^{15} \mathrm{~N}$ NMR $\left(\mathrm{CDCl}_{3}, 40 \mathrm{MHz}\right) \delta \mathrm{ppm}: 109.6(\mathrm{~N}-4), 172.6(\mathrm{~N}-9)$. Anal. Calcd. for $\mathrm{C}_{20} \mathrm{H}_{16} \mathrm{~N}_{2} \mathrm{O}_{2}$ (316.35): C, 75.93; H, 5.10; N, 8.85\%. Found: C, 78.88; H, 5.04; N, 8.92\%.

\section{Ethyl 1-acetyl-4-benzyl-4H-pyrrolo[1,2-a]benzimidazole-3-carboxylate (3c)}

Orange crystals, mp 168-169 ${ }^{\circ} \mathrm{C}$. FT-IR $\left(\mathrm{KBr}, \mathrm{cm}^{-1}\right)$ : 2979, 1688, 1629, 1572, 1501, 1475, 1391, 1367, 1305, 1236, 1182, 1146, 1092, 1062. ${ }^{1} \mathrm{H} \mathrm{NMR}\left(\mathrm{CDCl}_{3}, 400 \mathrm{MHz}\right) \delta \mathrm{ppm}: 1.34$ (t, 3H, J = 7.1 Hz, $\mathrm{CH}_{3}-\mathrm{Et}$ ), 2.55 (s, 3H, $\mathrm{CH}_{3} \mathrm{CO}$ ), 4.30 (q, 2H, J 7.1 Hz, CH $\left.2-\mathrm{Et}\right), 6.08$ (s, 2H, $\mathrm{CH}_{2}-\mathrm{N}$ ), 7.20-7.32 (m, 8H, 
benzene rings), $7.74(\mathrm{~s}, 1 \mathrm{H}, \mathrm{H}-2), 8.98(\mathrm{~d}, 1 \mathrm{H}, J 8.1 \mathrm{~Hz}, \mathrm{H}-8)$. The individual chemical shifts, multiplicities and coupling constants for the 7.20-7.32 multiplet were obtained from undecoupled HSQC as follows: 7.20 (d, 2H, J 7.3 Hz, H-2'), 7.220 (d, 1H, J $8.0 \mathrm{~Hz}, \mathrm{H}-5), 7.222$ (t, 1H, J 7.2 Hz, H-4'), $7.260(\mathrm{t}, 1 \mathrm{H}, J 8.1 \mathrm{~Hz}, \mathrm{H}-7), 7.263\left(\mathrm{t}, 2 \mathrm{H}, J 7.5 \mathrm{~Hz}, \mathrm{H}-3\right.$ '), $7.29(\mathrm{t}, 1 \mathrm{H}, J 8.1 \mathrm{~Hz}, \mathrm{H}-6) .{ }^{13} \mathrm{C} \mathrm{NMR}\left(\mathrm{CDCl}_{3}\right.$, $100 \mathrm{MHz}) \delta$ ppm: $14.5\left(\mathrm{CH}_{3}-\mathrm{Et}\right), 25.8\left(\mathrm{CH}_{3} \mathrm{CO}\right), 48.5\left(\mathrm{CH}_{2}-\mathrm{N}\right), 60.1\left(\mathrm{CH}_{2}-\mathrm{Et}\right), 92.5(\mathrm{C}-3), 110.1(\mathrm{CH}-$ 5), 117.3 (CH-8), 121.5 (CH-7), 122.0 (C-1), 124.3 (CH-6), 126.7 (CH-2'), 127.4 (CH-2 and C-8a), 127.6 (CH-4'), 128.8 (CH-3'), 136.2 (C-4a), 136.7 (C-1'), $143.5(\mathrm{C}-3 \mathrm{a}), 163.4(\mathrm{COO}), 185.1(\mathrm{CO}) .{ }^{15} \mathrm{~N}$ NMR (CDCl3, $40 \mathrm{MHz}) \delta$ ppm: 116.7 (N-4), 171.5 (N-9). Anal. Calcd. for $\mathrm{C}_{22} \mathrm{H}_{20} \mathrm{~N}_{2} \mathrm{O}_{3}$ (360.41): C, 73.31; H, 5.59; N, 7.77\%. Found: C, 73.39; H, 5.66; N, 7.69\%.

\section{1-Acetyl-3-benzoyl-4-benzyl-4H-pyrrolo[1,2-a]benzimidazole (3d)}

Pale yellow crystals, mp 182-184 ${ }^{\circ} \mathrm{C}$. FT-IR $\left(\mathrm{KBr}, \mathrm{cm}^{-1}\right)$ : 3030, 1648, 1618, 1544, 1503, 1448, 1390, 1367, 1289, 1195, 1158, 1066. ${ }^{1} \mathrm{H} \mathrm{NMR}\left(\mathrm{CDCl}_{3}, 400 \mathrm{MHz}\right) \delta \mathrm{ppm}: 2.48$ (s, 3H, $\left.\mathrm{CH}_{3} \mathrm{CO}\right), 6.19$ (s, 2H, $\left.\mathrm{CH}_{2}-\mathrm{N}\right), 7.18-7.37(\mathrm{~m}, 8 \mathrm{H}$, benzene rings), $7.46(\mathrm{~s}, 1 \mathrm{H}, \mathrm{H}-2), 7.51$ (t, $2 \mathrm{H}, J 7.4 \mathrm{~Hz}, \mathrm{H}-3$ "), $7.58(\mathrm{t}, 1 \mathrm{H}, J$ $7.3 \mathrm{~Hz}, \mathrm{H}-4$ "), 7.84 (d, 2H, J 7.1 Hz, H-2"), 9.02 (d, 1H, J 7.8 Hz, H-8). The individual chemical shifts, multiplicities and coupling constants for the 7.18-7.41 multiplet were obtained from undecoupled HSQC as follows: $7.23\left(\mathrm{t}, 1 \mathrm{H}, J 7.1 \mathrm{~Hz}, \mathrm{H}-4^{\prime}\right), 7.24$ (d, 2H, J 8.0 Hz, H-2'), 7.26 (d, 2H, J 8.0 Hz, H-3'), 7.35 $(\mathrm{t}, 1 \mathrm{H}, J 8.1 \mathrm{~Hz}, \mathrm{H}-7), 7.39$ (t, $1 \mathrm{H}, J 8.0 \mathrm{~Hz}, \mathrm{H}-6), 7.40$ (d, 1H, $J 8.1 \mathrm{~Hz}, \mathrm{H}-5) .{ }^{13} \mathrm{C} \mathrm{NMR}\left(\mathrm{CDCl}_{3}, 100\right.$ MHz) $\delta$ ppm: $25.8\left(\mathrm{CH}_{3}\right), 48.9\left(\mathrm{CH}_{2}-\mathrm{N}\right), 102.1(\mathrm{C}-3), 110.5(\mathrm{CH}-5), 117.6(\mathrm{CH}-8), 121.9(\mathrm{CH}-7), 122.2$ (C-1), 124.7 (CH-6), 126.9 (CH-2'), 127.3 (C-8a), 127.6 (CH-4'), 128.3 (CH-3”), 128.7 (CH-3'), 129.0 (CH-2), 129.1 (CH-2”), 131.5 (CH-4”), 136.3 (C-4a), 136.5 (C-1'), 140.1 (C-1”), 144.4 (C-3a), 185.3 $\left(\mathrm{COCH}_{3}\right), 188.8(\mathrm{CO}-\mathrm{Ph}) .{ }^{15} \mathrm{~N}$ NMR $(\mathrm{CDCl} 3,40 \mathrm{MHz}) \delta \mathrm{ppm}: 122.6(\mathrm{~N}-4), 172.9(\mathrm{~N}-9)$. Anal. Calcd. for $\mathrm{C}_{26} \mathrm{H}_{20} \mathrm{~N}_{2} \mathrm{O}_{2}$ (392.45): C, 79.57; H, 5.14; N, 7.14\%. Found: C, 79.66; H, 5.21; N, 7.09\%.

\section{Dimethyl 1-acetyl-4-benzyl-4H-pyrrolo[1,2-a]benzimidazole-2,3-dicarboxylate (3e)}

Pink crystals, mp $179-181^{\circ} \mathrm{C}$. FT-IR $\left(\mathrm{KBr}, \mathrm{cm}^{-1}\right)$ : 2951, 1732, 1701, 1636, 1573, 1500, 1464, 1380, 1332, 1263, 1218, 1171, 1095. ${ }^{1} \mathrm{H}$ NMR $\left(\mathrm{CDCl}_{3}, 400 \mathrm{MHz}\right) \delta$ ppm: 2.49 (s, 3H, $\left.\mathrm{CH}_{3} \mathrm{CO}\right), 3.76(\mathrm{~s}, 3 \mathrm{H}$, $\left.\mathrm{CH}_{3} \mathrm{COO}-3\right), 4.02$ (s, 3H, $\left.\mathrm{CH}_{3} \mathrm{COO}-2\right), 6.06$ (s, 2H, $\left.\mathrm{CH}_{2}-\mathrm{N}\right), 7.17$ (d, 2H, J 7.9 Hz, H-2'), 7.21-7.29 (m, $5 \mathrm{H}$, benzene rings), 7.33 (td, $1 \mathrm{H}, J 7.6 \mathrm{~Hz}, 0.3 \mathrm{~Hz}, \mathrm{H}-6), 8.93(\mathrm{~d}, 1 \mathrm{H}, J 7.8 \mathrm{~Hz}, \mathrm{H}-8)$. The individual chemical shifts, multiplicities and coupling constants for the 7.21-7.29 multiplet were obtained from undecoupled HSQC as follows: $7.24(\mathrm{t}, 1 \mathrm{H}, J 7.6 \mathrm{~Hz}, \mathrm{H}-4$ ') $7.26(\mathrm{~d}, 1 \mathrm{H}, J 8.3 \mathrm{~Hz}, \mathrm{H}-5), 7.280(\mathrm{t}, 1 \mathrm{H}, J$ $8.0 \mathrm{~Hz}, \mathrm{H}-7), 7.282$ (t, 2H, J 7.6 Hz, H-3'). ${ }^{13} \mathrm{C} \mathrm{NMR}\left(\mathrm{CDCl}_{3}, 100 \mathrm{MHz}\right) \delta \mathrm{ppm}: 27.2\left(\mathrm{CH}_{3} \mathrm{CO}\right), 48.5$ $\left(\mathrm{CH}_{2}-\mathrm{N}\right), 51.7\left(\mathrm{CH}_{3} \mathrm{COO}-3\right), 53.1\left(\mathrm{CH}_{3} \mathrm{COO}-2\right), 90.6(\mathrm{C}-3), 110.0(\mathrm{CH}-5), 117.9(\mathrm{CH}-8), 119.4(\mathrm{C}-1)$, 121.7 (CH-7), 124.9 (CH-6), 126.5 (CH-2'), 127.1 (C-8a), 127.7 (CH-4'), 128.8 (CH-3'), 130.9 (C-2), 136.4 (C-1'), 136.5 (C-4a), 141.8 (C-3a), 162.6 (COO-3), 166.9 (COO-2), 185.3 (CO). ${ }^{15} \mathrm{~N}$ NMR $(\mathrm{CDCl} 3,40 \mathrm{MHz}) \delta$ ppm: $116.6(\mathrm{~N}-4), 170.6(\mathrm{~N}-9)$. Anal. Calcd. for $\mathrm{C}_{23} \mathrm{H}_{20} \mathrm{~N}_{2} \mathrm{O}_{5}$ (404.41): $\mathrm{C}, 68.31 ; \mathrm{H}$, $4.98 ; \mathrm{N}, 6.93 \%$. Found: C, 68.25; H, 5.04; N, 7.01\%.

\section{Dimethyl 1-acetyl-4-(4-methylbenzyl)-4H-pyrrolo[1,2-a]benzimidazole-2,3-dicarboxylate (3f)}

Light yellow crystals, $\mathrm{mp} 143-145^{\circ} \mathrm{C}$. FT-IR $\left(\mathrm{KBr}, \mathrm{cm}^{-1}\right)$ : 1733, 1703, 1636, 1577, 1501, 1466, 1379, 1333, 1218, 1172, 1094. ${ }^{1} \mathrm{H} \mathrm{NMR}\left(\mathrm{CDCl}_{3}, 400 \mathrm{MHz}\right) \delta \mathrm{ppm}: 2.32$ (s, 3H, $\left.\mathrm{CH}_{3}-4^{\prime}\right), 2.53$ (s, 3H, $\left.\mathrm{CH}_{3} \mathrm{CO}\right)$, 3.82 (s, 3H, CH 3 COO-3), 4.05 (s, 3H, $\left.\mathrm{CH}_{3} \mathrm{COO}-2\right), 6.02$ (s, 2H, $\mathrm{CH}_{2}-\mathrm{N}$ ), 7.11 (bs, 4H, H-2' and H-3'), 7.29-7.32 (m, 2H, benzene ring), 7.37 (td, $1 \mathrm{H}, J 7.6 \mathrm{~Hz}, 1.0 \mathrm{~Hz}, \mathrm{H}-6), 8.97$ (dd, 1H, J 8.0 Hz, 1.1 Hz, H8).The individual chemical shifts, multiplicities and coupling constants for the broad singlet at $7.11 \mathrm{ppm}$ and 7.21-7.29 multiplet were obtained from undecoupled HSQC as follows: 7.09 (d, 2H, $J$ 8.4 Hz, H2'), 7.10 (d, 2H, J 8.4 Hz, H-3'), 7.29 (d, 1H, J 8.3 Hz, H-5), 7.30 (t, 1H, J 8.0 Hz, H-7). ${ }^{13} \mathrm{C}$ NMR $\left(\mathrm{CDCl}_{3}, 100 \mathrm{MHz}\right) \delta \mathrm{ppm}: 21.0\left(\mathrm{CH}_{3}-4\right), 27.2\left(\mathrm{CH}_{3} \mathrm{CO}\right), 48.3\left(\mathrm{CH}_{2}-\mathrm{N}\right), 51.7\left(\mathrm{CH}_{3} \mathrm{COO}-3\right), 53.1$ ( $\left.\mathrm{CH}_{3} \mathrm{COO}-2\right), 90.6$ (C-3), $110.1(\mathrm{CH}-5), 117.9(\mathrm{CH}-8), 119.4$ (C-1), $121.7(\mathrm{CH}-7), 124.9(\mathrm{CH}-6), 126.5$ (CH-2'), 127.1 (C-8a), 129.4 (CH-3'), 130.9 (C-2), 133.4 (C-1'), 136.5 (C-4a), 137.4 (C-4'), 141.8 (C- 


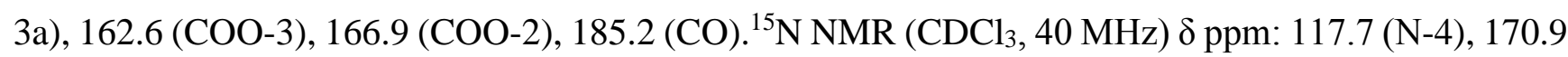
(N-9). Anal. Calcd. for $\mathrm{C}_{24} \mathrm{H}_{22} \mathrm{~N}_{2} \mathrm{O}_{5}$ (418.44): C, 68.89; H, 5.30; N, 6.69\%. Found: C, 68.96; H, 5.35; N, $6.62 \%$.

\section{Ethyl 4-methylene-5-benzyl-4,5-dihydropyrrolo[1,2-a]quinoxalin-2-carboxylate (4c)}

Yellow crystals, mp 156-158 ${ }^{\circ} \mathrm{C}$. FT-IR $\left(\mathrm{KBr}, \mathrm{cm}^{-1}\right)$ : 3137, 2980, 1697, 1618, 1557, 1528, 1472 , 1365, 1307, 1270, 1227, 1205, 1158, 1029. ${ }^{1} \mathrm{H} \mathrm{NMR}\left(\mathrm{CDCl}_{3}, 400 \mathrm{MHz}\right) \delta \mathrm{ppm}: 1.39$ (t, 3H, J 7.1 Hz, $\mathrm{CH}_{3}$-Et), 3.96 (d, 1H, J $2.0 \mathrm{~Hz},=\mathrm{CH}_{2}$ A-cis to N-5), 4.35 (d, 2H, J 7.2 Hz, $\left.\mathrm{CH}_{2}-\mathrm{Et}\right), 4.55$ (d, $1 \mathrm{H}, J 2.1$ $\mathrm{Hz},=\mathrm{CH}_{2}$ B-trans to N-5), $5.02\left(\mathrm{~s}, 2 \mathrm{H}, \mathrm{CH}_{2}-\mathrm{N}\right), 6.77(\mathrm{~d}, 1 \mathrm{H}, J 8.3 \mathrm{~Hz}, \mathrm{H}-6), 6.92(\mathrm{t}, 1 \mathrm{H}, J 7.3 \mathrm{~Hz}, \mathrm{H}-8)$, $6.95(\mathrm{~d}, 1 \mathrm{H}, J 1.4 \mathrm{~Hz}, \mathrm{H}-3), 7.06(\mathrm{t}, 1 \mathrm{H}, J 7.3 \mathrm{~Hz}, \mathrm{H}-7), 7.25$ (d, 2H, $J 8.4 \mathrm{~Hz}, \mathrm{H}-2$ ', partially overlapped with $\mathrm{CDCl}_{3}$ ), 7.27 (t, $1 \mathrm{H}, J 7.1 \mathrm{~Hz}, \mathrm{H}-4$ '), 7.35 (t, 2H, J 7.5 Hz, H-3'), 7.50 (d, 1H, J 7.9 Hz, H-9), 7.97 $(\mathrm{d}, 1 \mathrm{H}, J 1.2 \mathrm{~Hz}, \mathrm{H}-1) .{ }^{13} \mathrm{C} \mathrm{NMR}\left(\mathrm{CDCl}_{3}, 100 \mathrm{MHz}\right) \delta \mathrm{ppm}: 14.5\left(\mathrm{CH}_{3}-\mathrm{Et}\right), 51.0\left(\mathrm{CH}_{2}-\mathrm{N}\right), 60.2\left(\mathrm{CH}_{2}-\right.$ $\mathrm{Et}), 80.0\left(=\mathrm{CH}_{2}\right), 105.7(\mathrm{CH}-3), 113.9(\mathrm{CH}-6), 114.5(\mathrm{CH}-9), 117.7(\mathrm{CH}-1), 119.2(\mathrm{C}-2), 119.8(\mathrm{CH}-8)$, 122.9 (C-9a), 126.1 (CH-2'), 126.5 (CH-7), 126.6 (C-3a), 127.1 (CH-4'), 128.9 (CH-3'), 132.7 (C-5a), 135.9 (C-1'), 137.0 (C-4), 164.5 (COO). ${ }^{15} \mathrm{~N}$ NMR (CDCl3, $\left.40 \mathrm{MHz}\right) \delta$ ppm: $91.4(\mathrm{~N}-5), 166.2(\mathrm{~N}-10)$. Anal. Calcd. for $\mathrm{C}_{22} \mathrm{H}_{20} \mathrm{~N}_{2} \mathrm{O}_{2}$ (344.41): C, 76.72; H, 5.85; N, 8.13\%. Found: C, 76.65; H, 5.81; N, 8.11\%.

\section{Dimethyl 4-methylene-5-benzyl-4,5-dihydropyrrolo[1,2-a]quinoxalin-2,3-dicarboxylate (4e)}

Beige crystals, mp $173-174{ }^{\circ} \mathrm{C}$. FT-IR $\left(\mathrm{KBr}, \mathrm{cm}^{-1}\right): 3141,1725,1705,1613,1273,1247,1197 .{ }^{1} \mathrm{H}-$ NMR $\left(300 \mathrm{MHz}, \mathrm{CDCl}_{3}\right) \delta \mathrm{ppm}: 3.86,3.94\left(2 \mathrm{~s}, 6 \mathrm{H}, 2 \mathrm{xCH}_{3} \mathrm{O}\right), 4.13\left(\mathrm{~d}, 1 \mathrm{H}, J=3.1 \mathrm{~Hz},=\mathrm{CH}_{2}\right.$ A-cis to N5), $4.25\left(\mathrm{~d}, 1 \mathrm{H}, J 3.1 \mathrm{~Hz},=\mathrm{CH}_{2}\right.$ B-trans to N-5), $4.99\left(\mathrm{~s}, 2 \mathrm{H}, \mathrm{CH}_{2}-\mathrm{N}\right), 6.74(\mathrm{~d}, 1 \mathrm{H}, \mathrm{H}-6), 6.92(\mathrm{t}, 1 \mathrm{H}, \mathrm{H}-$ 8), 7.07 (t, 1H, H-7), 7.21 (d, 2H, H-2'), 7.27 (t, 1H, H-4' partially overlapped with $\left.\mathrm{CDCl}_{3}\right), 7.32(\mathrm{t}, 2 \mathrm{H}$, $\mathrm{H}-3$ ') 7.47 (d, 1H, H-9), 7.92 (s, 1H, H-1). ${ }^{13} \mathrm{C}-\mathrm{NMR}\left(75 \mathrm{MHz}, \mathrm{CDCl}_{3}\right) \delta \mathrm{ppm}: 51.2\left(\mathrm{CH}_{2}-\mathrm{N}\right), 51.8$, $52.9\left(2 \mathrm{xCH}_{3} \mathrm{O}\right), 84.9\left(=\mathrm{CH}_{2}\right), 114.0(\mathrm{C}-3), 114.2(\mathrm{CH}-6), 114.7(\mathrm{CH}-9), 117.3(\mathrm{C}-2), 117.7(\mathrm{CH}-1)$, 119.9 (CH-8), 122.4 (C-9a), 124.0 (C-3a), 126.1 (CH-2'), 127.1 (CH-7), 127.2 (CH-4'), 128.9 (CH-3'), 132.8 (C-5a), 135.6 (C-1'), 135.8 (C-4), 163.5, 167.2 (2xCOO). Anal. Calcd. for $\mathrm{C}_{23} \mathrm{H}_{20} \mathrm{~N}_{2} \mathrm{O}_{4}(388.42)$ : C, 71.12; H, 5.19; N, 7.21\%. Found: C, 71.20; H, 5.22; N, 7.16\%.

\section{Dimethyl 4-methylene-5-(4-methylbenzyl)-4,5-dihydropyrrolo[1,2-a]quinoxalin-2,3-dicarboxylate (4f)}

Beige crystals, mp $163-165^{\circ} \mathrm{C}$. FT-IR $\left(\mathrm{KBr}, \mathrm{cm}^{-1}\right): 3141,2949,1710,1616,1532,1437,1406,1362$, 1275, 1198, 1164, 1063. ${ }^{1} \mathrm{H} \mathrm{NMR}\left(\mathrm{CDCl}_{3}, 400 \mathrm{MHz}\right) \delta \mathrm{ppm}: 2.33\left(\mathrm{~s}, 3 \mathrm{H}, \mathrm{CH}_{3}-4^{\prime}\right), 3.86\left(\mathrm{~s}, 3 \mathrm{H}, \mathrm{CH}_{3}{ }^{-}\right.$ 2), $3.94\left(\mathrm{~s}, 3 \mathrm{H}, \mathrm{CH}_{3}-3\right), 4.14\left(\mathrm{~d}, 1 \mathrm{H}, J 3.0 \mathrm{~Hz},=\mathrm{CH}_{2} A\right.$-cis to $\left.\mathrm{N}-5\right), 4.54\left(\mathrm{~d}, 1 \mathrm{H}, J 3.0 \mathrm{~Hz},=\mathrm{CH}_{2} B\right.$-trans to N-5), $4.96\left(\mathrm{bs}, 2 \mathrm{H}, \mathrm{CH}_{2}-\mathrm{N}\right), 6.74(\mathrm{~d}, 1 \mathrm{H}, J 8.1 \mathrm{~Hz}, \mathrm{H}-6), 6.92(\mathrm{t}, 1 \mathrm{H}, J 7.4 \mathrm{~Hz}, \mathrm{H}-8), 7.07(\mathrm{t}, 1 \mathrm{H}, J 7.3 \mathrm{~Hz}$, H-7), 7.10 (d, 2H, J 8.1 Hz, H-2'), 7.15 (d, 2H, J 8.0 Hz, H-3'), 7.47 (d, 1H, J 7.2 Hz, H-6), 7.92 (s, 1H, $\mathrm{H}-1) .{ }^{13} \mathrm{C}$ NMR $\left(\mathrm{CDCl}_{3}, 100 \mathrm{MHz}\right) \delta \mathrm{ppm}: 21.0\left(\mathrm{CH}_{3}-4\right), 51.5\left(\mathrm{CH}_{2}-\mathrm{N}\right), 51.7\left(\mathrm{CH}_{3}-2\right), 52.9\left(\mathrm{CH}_{3}-3\right)$, $84.8\left(=\mathrm{CH}_{2}\right), 113.9(\mathrm{C}-3), 114.2(\mathrm{CH}-6), 114.7(\mathrm{CH}-9), 117.3(\mathrm{C}-2), 117.6(\mathrm{CH}-1), 119.8(\mathrm{CH}-8), 122.2$ (C-9a), 124.0 (C-3a), 126.0 (CH-2'), 127.1 (CH-7), 129.6 (CH-3'), 132.4 (C-1'), 132.9 (C-5a), 135.7 (C-4), 136.8 (C-4'), 163.5 (COO-2), 167.2 (COO-3). ${ }^{15} \mathrm{~N}$ NMR $\left(\mathrm{CDCl}_{3}, 40 \mathrm{MHz}\right) \delta$ ppm: 93.4 (N-5), $167.1(\mathrm{~N}-10)$. Anal. Calcd. for $\mathrm{C}_{24} \mathrm{H}_{22} \mathrm{~N}_{2} \mathrm{O}_{4}$ (402.44): C, 71.63; H, 5.51; N, 6.96\%. Found: C, 71.59; H, $5.44 ; \mathrm{N}, 7.01 \%$.

\section{Ethyl 4-oxo-5-methyl-5H-pyrrolo[1,2-a]quinoxalin-2-carboxylate (5a)}

Pale yellow crystals, mp 191-193 ${ }^{\circ} \mathrm{C}$. FT-IR $\left(\mathrm{KBr}, \mathrm{cm}^{-1}\right)$ : 2976, 2897, 1706, 1653, 1555, 1520, 1476, 1362, 1341, 1306, 1269, 1190, 1083, 1028. ${ }^{1} \mathrm{H} \mathrm{NMR}\left(\mathrm{CDCl}_{3}, 400 \mathrm{MHz}\right) \delta \mathrm{ppm}: 1.39(\mathrm{t}, 3 \mathrm{H}, J \mathrm{~J} .2 \mathrm{~Hz}$, $\mathrm{CH}_{3}$-Et), 3.64 (s, 3H, CH $)_{3}, 4.35$ (q, 2H, J 7.1 Hz, CH 2 -Et), 7.26 (t, 1H, J 7.6 Hz, H-8), 7.32 (d, 1H, J $7.2 \mathrm{~Hz}, \mathrm{H}-6), 7.39$ (t, 1H, J 7.2 Hz, H-7), 7.57 (d, 1H, J 1.5 Hz, H-3), 7.71 (d, 1H, J 7.1 Hz, H-9), 8.17 $(\mathrm{d}, 1 \mathrm{H}, J 1.5 \mathrm{~Hz}, \mathrm{H}-1) .{ }^{13} \mathrm{C} \mathrm{NMR}\left(\mathrm{CDCl}_{3}, 100 \mathrm{MHz}\right) \delta \mathrm{ppm}: 14.4\left(\mathrm{CH}_{3}-\mathrm{Et}\right), 28.5\left(\mathrm{CH}_{3}\right), 60.5\left(\mathrm{CH}_{2}-\mathrm{Et}\right)$, 113.4 (CH-3), 114.8 (CH-9), 115.9 (CH-6), 119.2 (CH-1), 120.2 (C-2), 123.1 (CH-8), 123.2 (C-9a), 
123.8 (C-3a), 126.8 (CH-7), 130.8 (C-5a), 155.2 (C-4), 163.8 (COO). ${ }^{15} \mathrm{~N}$ NMR (CDCl3, $\left.40 \mathrm{MHz}\right) \delta$ ppm: $126.1(\mathrm{~N}-5), 173.2(\mathrm{~N}-10)$. Anal. Calcd. for $\mathrm{C}_{15} \mathrm{H}_{14} \mathrm{~N}_{2} \mathrm{O}_{3}(270.28)$ : C, 66.66; H, 5.22; N, 10.36\%. Found: C, 66.73; H, 5.27; N, 10.29\%.

\section{2-Benzoyl-4-oxo-5-methyl-5H-pyrrolo[1,2-a]quinoxaline (5b)}

Orange crystals, mp $211-213^{\circ} \mathrm{C}$. FT-IR $\left(\mathrm{KBr}, \mathrm{cm}^{-1}\right): 3121,3054,1641,1627,1544,1518,1476$, 1346, 1308, 1282, 1146. ${ }^{1} \mathrm{H}$ NMR $\left(\mathrm{CDCl}_{3}, 400 \mathrm{MHz}\right) \delta \mathrm{ppm}: 3.67\left(\mathrm{~s}, 3 \mathrm{H}, \mathrm{CH}_{3}\right), 7.28(\mathrm{t}, 1 \mathrm{H}, J 8.2 \mathrm{~Hz}$, H-8), 7.35 (d, 1H, J 7.5 Hz, H-6), 7.41 (t, 1H, J 8.3 Hz, H-7), 7.51 (t, 2H, J 7.7 Hz, H-3'), 7.57 (d, 1H, $J 1.4 \mathrm{~Hz}, \mathrm{H}-3), 7.60$ (t, $\left.1 \mathrm{H}, J 7.4 \mathrm{~Hz}, \mathrm{H}-4^{\prime}\right), 7.76$ (d, 1H, $\left.J 7.5 \mathrm{~Hz}, \mathrm{H}-9\right), 7.92$ (d, 2H, J 7.2 Hz, H-2'), $8.25(\mathrm{~d}, 1 \mathrm{H}, J 1.5 \mathrm{~Hz}, \mathrm{H}-1) .{ }^{13} \mathrm{C} \mathrm{NMR}\left(\mathrm{CDCl}_{3}, 100 \mathrm{MHz}\right) \delta \mathrm{ppm}: 28.6\left(\mathrm{CH}_{3}\right), 114.4(\mathrm{CH}-3), 115.0(\mathrm{CH}-$ 9), 116.0 (CH-6), 120.0 (CH-1), 123.1 (C-9a), 123.3 (CH-8), 123.9 (C-3a), 127.0 (C-2), 127.1 (CH-7), 128.5 (CH-3') 129.2 (CH-2'), 130.9 (C-5a), 132.3 (CH-4'), 138.5 (C-1'), $155.3(\mathrm{C}-4), 190.4(\mathrm{CO}) .{ }^{15} \mathrm{~N}$ NMR (CDCl3, $40 \mathrm{MHz}) \delta$ ppm: $126.3(\mathrm{~N}-5), 174.5(\mathrm{~N}-10)$. Anal. Calcd. for $\mathrm{C}_{19} \mathrm{H}_{14} \mathrm{~N}_{2} \mathrm{O}_{2}$ (302.33): C, 75.48; H, 4.67; N, 9.26\%. Found: C, 75.56; H, 4.70; N, 9.18\%.

\section{Ethyl 4-oxo-5-benzyl-5H-pyrrolo[1,2-a]quinoxalin-2-carboxylate (5c)}

It was synthesized directly in the NMR tube by slow transformation when keeping a solution of $\mathbf{4 c}$ in $\mathrm{CDCl}_{3}$ for two weeks.

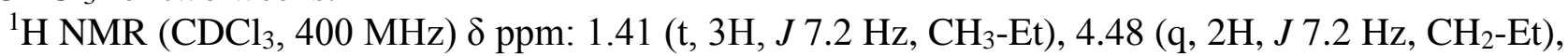
$5.51\left(\mathrm{~s}, 2 \mathrm{H}, \mathrm{CH}_{2}-\mathrm{N}\right), 7.21-7.36(\mathrm{~m}, 8 \mathrm{H}, \mathrm{H}-6,7,8$, aromatic rings), 7.68 (d, 1H, J 1.3 Hz, H-3), 7.73-7.75 (m, 1H, H-9), 8.25 (d, 1H, J $1.3 \mathrm{~Hz}, \mathrm{H}-1)$. The individual chemical shifts, multiplicities and coupling constants for the 7.21-7.29 multiplet were obtained from undecoupled HSQC as follows: 7.22 (t, $1 \mathrm{H}, J$ $8.4 \mathrm{~Hz}, \mathrm{H}-8$ ), 7.241 (t, 1H, J $8.0 \mathrm{~Hz}, \mathrm{H}-7), 7.242$ (d, 1H, J $8.1 \mathrm{~Hz}, \mathrm{H}-6), 7.25$ (t, 1H, J 7.3 Hz, H-4'),

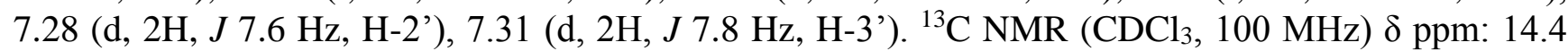
$\left(\mathrm{CH}_{3}-\mathrm{Et}\right), 45.2\left(\mathrm{CH}_{2}-\mathrm{N}\right), 60.6\left(\mathrm{CH}_{2}-\mathrm{Et}\right), 114.0(\mathrm{CH}-3), 115.0(\mathrm{CH}-9), 116.9(\mathrm{CH}-6), 119.4(\mathrm{CH}-1), 120.5$ (C-2), 123.3 (CH-8), 123.5 (C-9a), 123.7 (C-3a), 126.6 (CH-2'), 126.8 (CH-7), 127.5 (CH-4'), 128.9 (CH-3'), 130.1 (C-5a), 136.1 (C-1'), 155.5 (C-4), 163.8 (COO). ${ }^{15} \mathrm{~N}$ NMR (CDCl3, $\left.40 \mathrm{MHz}\right) \delta$ ppm: $175.5(\mathrm{~N}-10)$. The NMR data are consistent with the structure of $\mathbf{5 c}$ which was isolated and fully characterized from another reaction [27].

\section{2-Benzoyl-4-oxo-5-benzyl-5H-pyrrolo[1,2-a]quinoxaline (5d)}

White crystals, $\mathrm{mp} 222-223^{\circ} \mathrm{C}$. FT-IR $\left(\mathrm{KBr}, \mathrm{cm}^{-1}\right): 3122,1662,1633,1610,1542,1517,1472,1382$, 1358, 1279, 1220, 1178, 1132. ${ }^{1} \mathrm{H}$ NMR $\left(\mathrm{CDCl}_{3}, 400 \mathrm{MHz}\right) \delta \mathrm{ppm}$ : 5.52 (s, 2H, $\left.\mathrm{CH}_{2}-\mathrm{N}\right), 7.20-7.37$ (m, $8 \mathrm{H}, \mathrm{H}-6,7,8$, aromatic rings), 7.52 (t, 2H, J 7.2 Hz, H-3'), 7.61 (t, 1H, J 7.4 Hz, H-4'), 7.68 (d, 1H, J 1.6 Hz, H-3), 7.77 (d, 1H, J 6.6 Hz, H-9), 7.95 (d, 2H, J 7.1 Hz, H-2'), 8.31 (d, 1H, J 1.6 Hz, H-1). The individual chemical shifts, multiplicities and coupling constants for the 7.20-7.37 multiplet were obtained from undecoupled HSQC as follows: 7.24 (t, 1H, J 8.4 Hz, H-8), 7.26 (t, 1H, J 7.3 Hz, H-4"), 7.264 (t, 1H, J 8.0 Hz, H-7), 7.27 (d, 1H, J 8.4 Hz, H-6), 7.30 (d, 2H, J 7.7 Hz, H-2"), 7.33 (t, 2H, J 7.9 $\mathrm{Hz}, \mathrm{H}-3 ") .{ }^{13} \mathrm{C} \mathrm{NMR}\left(\mathrm{CDCl}_{3}, 100 \mathrm{MHz}\right) \delta \mathrm{ppm}: 45.2\left(\mathrm{CH}_{2}-\mathrm{N}\right), 114.9(\mathrm{CH}-3), 115.2(\mathrm{CH}-9), 116.9(\mathrm{CH}-$ 6), 120.2 (CH-1), 123.2 (C-9a), 123.4 (CH-8), 123.6 (C-3a), 126.6 (CH-2”), 127.1 (CH-7), 127.2 (C-2), 127.5 (CH-4”), 128.5 (CH-3'), 128.9 (CH-3”), 129.3 (CH-2'), 130.0 (C-5a), 132.4 (CH-4'), 135.9 (C1”), 138.5 (C-1'), 155.7 (C-4), 190.4 (CO). ${ }^{15} \mathrm{~N}$ NMR (CDCl3, $\left.40 \mathrm{MHz}\right) \delta \mathrm{ppm}: 137.9$ (N-5), 175.7 (N10). Anal. Calcd. for $\mathrm{C}_{25} \mathrm{H}_{18} \mathrm{~N}_{2} \mathrm{O}_{2}$ (378.42): C, 79.35; H, 4.79; N, 7.40\%. Found: C, 79.44; H, 4.83; N, $7.31 \%$.

\section{Dimethyl 4-oxo-5-(4-methylbenzyl)pyrrolo[1,2-a]quinoxalin-2,3-dicarboxylate (5f)}

Beige crystals, $\mathrm{mp} 244-246^{\circ} \mathrm{C}$. FT-IR $\left(\mathrm{KBr}, \mathrm{cm}^{-1}\right)$ : 1748, 1716, 1661, 1614, 1522, 1438, 1371, 1270 , 1245, 1152, 1075. ${ }^{1} \mathrm{H}$ NMR $\left(\mathrm{CDCl}_{3}, 400 \mathrm{MHz}\right) \delta \mathrm{ppm}: 2.29$ (s, 3H, $\mathrm{CH}_{3}-4$ '), 3.90 (s, 3H, $\left.\mathrm{CH}_{3}-2\right), 4.04$ (s, 3H, $\left.\mathrm{CH}_{3}-3\right), 5.42$ (bs, 2H, $\left.\mathrm{CH}_{2}-\mathrm{N}\right), 7.10$ (d, 2H, J 8.1 Hz, H-3'), 7.15 (d, 2H, J 8.1 Hz, H-3'), 7.21- 
$7.27(\mathrm{~m}, 3 \mathrm{H}$, aromatic ring), $7.72(\mathrm{~d}, 1 \mathrm{H}, J 7.6 \mathrm{~Hz}, \mathrm{H}-9), 8.18(\mathrm{~s}, 1 \mathrm{H}, \mathrm{H}-1)$. The individual chemical shifts, multiplicities and coupling constants for the 7.21-7.27 multiplet were obtained from undecoupled HSQC as follows: $7.23(\mathrm{t}, 1 \mathrm{H}, J 8.3 \mathrm{~Hz}, \mathrm{H}-8), 7.26(\mathrm{~d}, 1 \mathrm{H}, J 8.3 \mathrm{~Hz}, \mathrm{H}-6), 7.27(\mathrm{t}, 1 \mathrm{H}, J 8.1 \mathrm{~Hz}, \mathrm{H}-7)$. ${ }^{13} \mathrm{C} \mathrm{NMR}\left(\mathrm{CDCl}_{3}, 100 \mathrm{MHz}\right) \delta \mathrm{ppm}: 21.1\left(\mathrm{CH}_{3}-4{ }^{\prime}\right), 45.0\left(\mathrm{CH}_{2}-\mathrm{N}\right), 52.1\left(\mathrm{CH}_{3}-2\right), 53.1\left(\mathrm{CH}_{3}-3\right), 115.1$ (CH-9), 117.1 (CH-6), 117.8 (C-2), 118.8 (CH-1), 121.1 (C-3a), 122.7 (C-9a), 123.5 (CH-8), 126.5(C3), 126.6 (CH-2'), 127.4 (CH-7), 129.6 (CH-3'), 129.9 (C-5a), 132.5 (C-1'), 137.2 (C-1'), 154.4 (C-4), 162.9 (COO-2), $165.5(\mathrm{COO}-3) .{ }^{15} \mathrm{~N}$ NMR $\left(\mathrm{CDCl}_{3}, 40 \mathrm{MHz}\right) \delta \mathrm{ppm:} 139.2(\mathrm{~N}-5), 173.3(\mathrm{~N}-10)$. Anal. Calcd. for $\mathrm{C}_{23} \mathrm{H}_{20} \mathrm{~N}_{2} \mathrm{O}_{5}$ (404.41): C, 68.31; H, 4.98; N, 6.93\%. Found: C, 68.40; H, 5.04; N, 6.86\%.

\section{4-(2-Carbethoxy-5-methyl-5H-pyrrolo[1,2-a]quinoxalin-4-ylidene)-but-2-enoic acid ethyl ester (6a)}

Orange crystals, mp 160-162 ${ }^{\circ} \mathrm{C}$. FT-IR $\left(\mathrm{KBr}, \mathrm{cm}^{-1}\right): 3134,2977,1697,1575,1550,1527,1471$, 1443, 1409, 1335, 1272, 1180, 1151, 1033. ${ }^{1} \mathrm{H}$ NMR (DMSO-d6, $\left.400 \mathrm{MHz}\right) \delta$ ppm: 1.25 (t, 3H, J 7.1 $\left.\mathrm{Hz}, \mathrm{CH}_{3}-14\right), 1.35$ (t, 3H, J 7.0 Hz, $\left.\mathrm{CH}_{3}-2\right), 3.44\left(3 \mathrm{H}, \mathrm{s}, \mathrm{CH}_{3}-\mathrm{N}\right), 4.16$ (q, 2H, J 7.1 Hz, $\left.\mathrm{CH}_{2}-14\right), 4.32$ $\left(\mathrm{q}, 2 \mathrm{H}, J 7.1 \mathrm{~Hz}, \mathrm{CH}_{2}-2\right), 5.50(\mathrm{~d}, 1 \mathrm{H}, J 11 \mathrm{~Hz}, \mathrm{H}-13$ trans to $H-12), 7.02(\mathrm{~d}, 1 \mathrm{H}, J 12 \mathrm{~Hz}, \mathrm{H}-11$ cis to $N-5), 7.08(\mathrm{~d}, 1 \mathrm{H}, J 1.2 \mathrm{~Hz}, \mathrm{H}-3), 7.09-7.12(\mathrm{~m}, 1 \mathrm{H}, \mathrm{H}-8), 7.32-7.34(\mathrm{~m}, 2 \mathrm{H}, \mathrm{H}-6$ and H-7), 7.44 (t, 1H, $J 11.4 \mathrm{~Hz}, \mathrm{H}-12$ trans to $H-11), 8.01(\mathrm{~d}, 1 \mathrm{H}, J 8.2 \mathrm{~Hz}, \mathrm{H}-9), 8.46(\mathrm{~d}, 1 \mathrm{H}, J 1.3 \mathrm{~Hz}, \mathrm{H}-1) .{ }^{13} \mathrm{C}$ NMR (DMSO-d6, $100 \mathrm{MHz}) \delta$ ppm: $14.3\left(2 \mathrm{xCH}_{3}-\mathrm{Et}\right), 34.7\left(\mathrm{CH}_{3}-\mathrm{N}\right), 58.7\left(\mathrm{CH}_{2}-14\right), 59.9\left(\mathrm{CH}_{2}-2\right), 99.9(\mathrm{CH}-$ 11), 109.7 (CH-13), 111.0 (CH-3), 115.0 (CH-6), 115.8 (CH-9), 119.0 (C-2), 120.4 (CH-1), 121.3 (CH8), 123.0 (C-9a), 123.4 (C-3a), 127.0 (CH-7), 131.9 (C-5a), 138.9 (C-4), 141.6 (CH-12), 163.0 (COO2), $166.8(\mathrm{COO}-14) .{ }^{15} \mathrm{~N} \mathrm{NMR}\left(\mathrm{CDCl}_{3}, 40 \mathrm{MHz}\right) \delta \mathrm{ppm}: 91.1(\mathrm{~N}-5), 169.7(\mathrm{~N}-10)$. Anal. Calcd. for $\mathrm{C}_{21} \mathrm{H}_{22} \mathrm{~N}_{2} \mathrm{O}_{4}$ (366.41): C, 68.84; H, 6.05; N, 7.65\%. Found: C, 68.92; H, 6.10; N, 7.61\%.

\section{4-(2-Benzoyl-5-methyl-5H-pyrrolo[1,2-a]quinoxalin-4-ylidene)-1-phenyl-but-2-en-1-one (6b)}

Red crystals, mp 219-221 ${ }^{\circ} \mathrm{C}$. FT-IR (KBr, cm $\left.{ }^{-1}\right)$ : 1628, 1513, 1473, 1392, 1352, 1267, 1196, 1107 , 1019. ${ }^{1} \mathrm{H}$ NMR (DMSO-d6, $\left.400 \mathrm{MHz}\right) \delta$ ppm: $3.54\left(3 \mathrm{H}, \mathrm{s}, \mathrm{CH}_{3}\right), 6.21(\mathrm{~d}, 1 \mathrm{H}, J 12.2 \mathrm{~Hz}, \mathrm{H}-13), 7.14(\mathrm{t}$, $1 \mathrm{H}, J 8.0 \mathrm{~Hz}, \mathrm{H}-8), 7.18$ (d, 1H, J 14.3 Hz, H-11), 7.24 (s, 1H, H-3), 7.36 (t, 1H, J 7.7 Hz, H-7), 7.40 (d, 1H, J 6.9 Hz, H-6), 7.52 (t, 2H, J 7.5 Hz, H-3”), 7.59 (t, 1H, J 7.1 Hz, H-4"), 7.61 (t, 2H, J 7.6 Hz, H3'), 7.71 (t, 1H, J 7.4 Hz, H-4'), 7.95 (d, 2H, J 7.7 Hz, H-2'), 7.98 (d, 2H, J 7.7 Hz, H-2”), 8.16 (d, 1H, $J 7.9 \mathrm{~Hz}, \mathrm{H}-9$ ), 8.29 (t, $1 \mathrm{H}, J 12.6 \mathrm{~Hz}, \mathrm{H}-12), 8.59$ (s, 1H, H-1). ${ }^{13} \mathrm{C}$ NMR (DMSO-d6, $\left.100 \mathrm{MHz}\right) \delta \mathrm{ppm}$ : $34.9\left(\mathrm{CH}_{3}-\mathrm{N}\right), 101.9(\mathrm{CH}-13), 111.9(\mathrm{CH}-3), 115.2(\mathrm{CH}-6), 116.0(\mathrm{CH}-9), 119.1(\mathrm{CH}-11), 121.6(\mathrm{CH}-$ 8), 121.7 (CH-1), 123.1 (C-9a), 123.9 (C-3a), 126.3 (C-2), 127.2 (CH-7), 127.6 (CH-2"), 128.5 (CH3”), 128.6 (CH-3'), 128.9 (CH-2'), 131.7 (C-5a), 131.9 (CH-4”), 132.2 (CH-4'), 138.4 (C-1'), 138.7 (C1"), 139.9 (C-4), 142.4 (CH-12), 188.1 (CO-14), 188.9 (CO-2). ${ }^{15} \mathrm{~N}$ NMR (DMSO-d6, $\left.40 \mathrm{MHz}\right) \delta$ ppm: $97.3(\mathrm{~N}-5), 171.6(\mathrm{~N}-10)$. Anal. Calcd. for $\mathrm{C}_{2} \mathrm{H}_{22} \mathrm{~N}_{2} \mathrm{O}_{2}$ (430.50): C, 80.91; H, 5.15; N, 6.51\%. Found: C, $80.97 ; \mathrm{H}, 5.19 ; \mathrm{N}, 6.47 \%$.

\section{4-(2-Benzoyl-5-benzyl-5H-pyrrolo[1,2-a]quinoxalin-4-ylidene)-1-phenyl-but-2-en-1-one (6d)}

Red crystals, mp 198-200 ${ }^{\circ} \mathrm{C}$. FT-IR $\left(\mathrm{KBr}, \mathrm{cm}^{-1}\right): 3119,1633,1546,1516,1470,1380,1268,1197$, 1175, 1118, 1018. ${ }^{1} \mathrm{H}$ NMR (DMSO-d6, $\left.400 \mathrm{MHz}\right) \delta \mathrm{ppm}: 5.34\left(2 \mathrm{H}, \mathrm{s}, \mathrm{CH}_{2}-\mathrm{N}\right), 6.05(\mathrm{~d}, 1 \mathrm{H}, J 12.1 \mathrm{~Hz}$, H-13), 7.01 (d, 1H, J 14.0 Hz, H-11), 7.07 (d, 1H, J 8.2 Hz, H-6), 7.13 (t, 1H, J 7.7 Hz, H-8), 7.25 (t, 1H, J 7.7 Hz, H-7), 7.32 (s, 1H, H-3), 7.28-7.31 (m, 3H, H-2"' and H-4"'), 7.41 (t, 2H, J 7.5 Hz, H-3"'), 7.47 (t, 2H, J 7.5 Hz, H-3"), 7.56 (t, 1H, J 7.4 Hz, H-4"), 7.63 (t, 2H, J 7.4 Hz, H-3'), 7.72 (t, 1H, J 7.4 Hz, H-4'), 7.94 (d, 2H, J 7.4 Hz, H-2”), 7.98 (d, 2H, J 7.4 Hz, H-2'), 8.24 (d, 1H, J 7.9 Hz, H-9), 8.32 $(\mathrm{t}, 1 \mathrm{H}, J 12.4 \mathrm{~Hz}, \mathrm{H}-12), 8.69$ (s, 1H, H-1). ${ }^{13} \mathrm{C}$ NMR (DMSO-d6, $\left.100 \mathrm{MHz}\right) \delta \mathrm{ppm}: 50.8\left(\mathrm{CH}_{2}-\mathrm{N}\right), 101.0$ (CH-13), 112.7 (CH-3), 115.5 (CH-6), 116.1 (CH-9), 118.8 (CH-11), $121.9(\mathrm{CH}-8), 122.0$ (CH-1), 123.1 (C-9a), 123.5 (C-3a), 126.1 (CH-2"”), 126.5 (C-2), 127.1 (CH-4"'), 127.2 (CH-7), 127.7 (CH-2"), 128.4 (CH-3"), 128.7 (CH-3'), 128.9 (CH-3"'), 129.0 (CH-2'), 130.8 (C-5a), 132.0 (CH-4”), 132.3 (CH-4'), 135.5 (C-1"”), 138.3 (C-1'), 138.5 (C-1”), 139.5 (C-4), 142.5 (CH-12), 187.9 (CO-14), 188.9 (CO-2). 
${ }^{15} \mathrm{~N}$ NMR (DMSO-d6, $\left.40 \mathrm{MHz}\right) \delta$ ppm: $104.7(\mathrm{~N}-5), 171.3(\mathrm{~N}-10)$. Anal. Calcd. for $\mathrm{C}_{35} \mathrm{H}_{26} \mathrm{~N}_{2} \mathrm{O}_{2}$ (506.59): C, 82.98; H, 5.17; N, 5.57\%. Found: C, 83.05; H, 5.13; N, 5.50\%.

\section{Results and discussions}

The reactions of $N$-acetonylbenzimidazolium chlorides $\mathbf{1}$, easily obtained from 1 -substituted benzimidazoles and chloroacetone, with various activated alkynes $\mathbf{2}$ such as ethyl propiolate, 1phenylpropynone and dimethyl acetylenedicarboxylate, in the presence of bases, led to a mixture of pyrrolo[1,2-a]benzimidazoles 3, 4-methylene-pyrrolo[1,2-a]quinoxaline derivatives 4 and pyrrolo[1,2a] quinoxalin-4-one derivatives 5 (Scheme 1).<smiles></smiles>

1

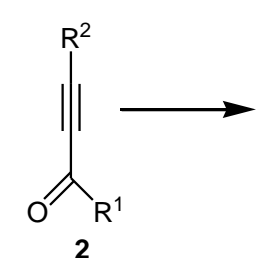<smiles></smiles>

3<smiles>[R]C(=O)c1cn2c(c1[R])C(=C)N([Z9])c1ccccc1-2</smiles>

4<smiles></smiles>

$$
\begin{aligned}
& \text { a: } \mathrm{R}=\mathrm{CH}_{3} ; \mathrm{R}^{1}=\mathrm{OC}_{2} \mathrm{H}_{5} ; \mathrm{R}^{2}=\mathrm{H} \\
& \text { b: } \mathrm{R}=\mathrm{CH}_{3} ; \mathrm{R}^{1}=\mathrm{C}_{6} \mathrm{H}_{5} ; \mathrm{R}_{2}=\mathrm{H} \\
& \text { c: } \mathrm{R}=\mathrm{CH}_{2} \mathrm{C}_{6} \mathrm{H}_{5} ; \mathrm{R}^{1}=\mathrm{OC}_{2} \mathrm{H}_{5} ; \mathrm{R}^{2}=\mathrm{H} \\
& \text { d: } \mathrm{R}=\mathrm{CH}_{2} \mathrm{C}_{6} \mathrm{H}_{5} ; \mathrm{R}^{1}=\mathrm{C}_{6} \mathrm{H}_{5} ; \mathrm{R}^{2}=\mathrm{H} \\
& \text { e: } \mathrm{R}=\mathrm{CH}_{2} \mathrm{C}_{6} \mathrm{H}_{5} ; \mathrm{R}^{1}=\mathrm{OCH}_{3} ; \mathrm{R}^{2}=\mathrm{CO}_{2} \mathrm{CH}_{3} \\
& \text { f: } \mathrm{R}=\mathrm{CH}_{2} \mathrm{C}_{6} \mathrm{H}_{4}-\mathrm{CH}_{3}(p) ; \mathrm{R}^{1}=\mathrm{OCH}_{3} ; \mathrm{R}^{2}=\mathrm{CO}_{2} \mathrm{CH}_{3} \\
& \text { g: } \mathrm{R}=\mathrm{CH}_{2} \mathrm{C}_{6} \mathrm{H}_{5} ; \mathrm{R}^{1}=\mathrm{CH}_{3} ; \mathrm{R}^{2}=\mathrm{H}
\end{aligned}
$$

Scheme 1. The reaction scheme for the 1,3-dipolar cycloaddition

Mild reaction conditions are required, implying the mixing of $N$-acetonylbenzimidazolium chlorides $\mathbf{1}$ with activated alkynes $\mathbf{2}$ in acetonitrile, in the presence of triethylamine, followed by heating of the reaction mixture at reflux temperature for 3 hours. The cold reaction mixtures were poured into water under stirring, extracted with $\mathrm{CHCl}_{3}$, dried and, after the removal of the solvent, the crude reaction products were chromatographed on $\mathrm{SiO}_{2}$ packed column by eluting with EtOAc:hexane $(1: 4 \mathrm{v} / \mathrm{v})$.

Numbering of atoms of the parent skeletons and substituents is exemplified in Figure 1.
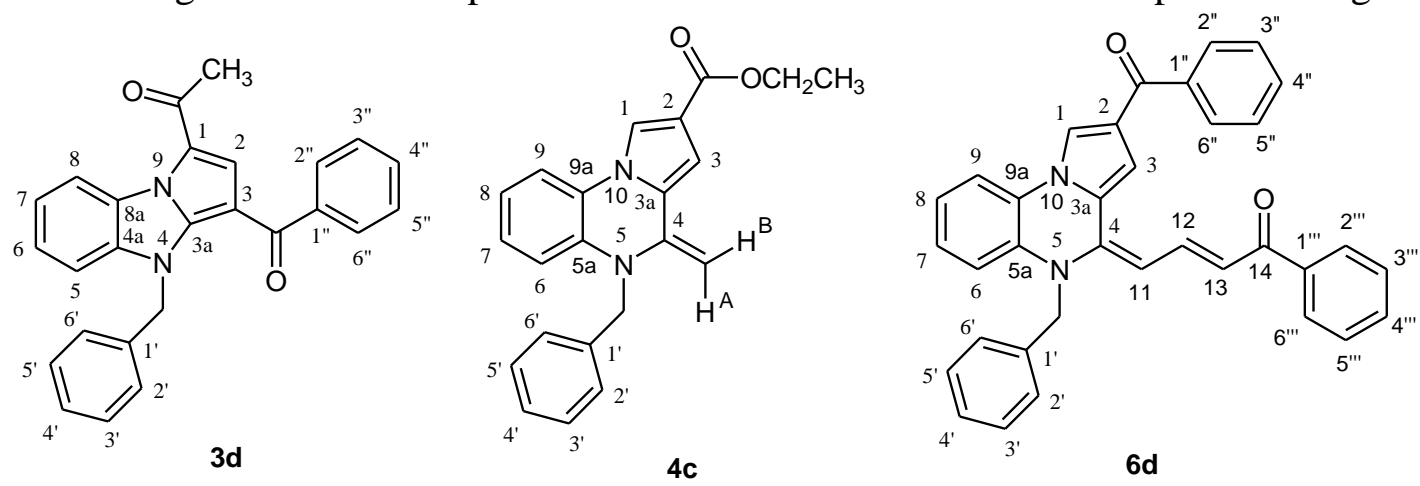

Figure 1. Numbering of atoms in $\mathbf{3 c}, \mathbf{4 c}$, and $\mathbf{6 d}$.

Samples of all crude reaction products were concentrated and analyzed by HPLC and the major components were identified. As pyrrolo[1,2-a]quinoxaline compounds of type $\mathbf{4}$ are very reactive, only $\mathbf{4 c}, \mathbf{4 e}$ and $\mathbf{4 f}$ were isolated from the reaction mixture and completely characterized. These enamine-type compounds $\mathbf{4}$ are easily transformed into pyrrolo[1,2-a]quinoxalin-4-one compounds $\mathbf{5}$, or react with a molecule of activated alkynes $\mathbf{2}$ to give addition reaction products $\mathbf{6}$ (Figure 2). 
<smiles>[R]c1c(C=O)cn2c1C(=CC=CC=O)N([2H])c1ccccc1-2</smiles>

Figure 2. General formula

for addition products 6

Compounds isolated and characterized from all these reactions are presented in Table 1.

The yields of pyrrolo[1,2-a]benzimidazoles 3, pyrrolo[1,2-a]quinoxaline derivatives 4-6 are influenced by the reaction conditions as well as the nature of the reagents (Table 2).

Table 1. The isolated reaction products with their characteristic melting points

\begin{tabular}{|c|c|c|c|c|c|c|c|}
\hline \multicolumn{7}{|c|}{ Isolated compounds } \\
\hline Compd. & $\mathrm{mp}\left({ }^{\circ} \mathrm{C}\right)$ & Compd. & $\mathrm{mp}\left({ }^{\circ} \mathrm{C}\right)$ & Compd. & $\mathrm{mp}\left({ }^{\circ} \mathrm{C}\right)$ & Compd. & $\mathrm{mp}\left({ }^{\circ} \mathrm{C}\right)$ \\
\hline $\mathbf{3 a}$ & $173-174$ & - & - & $\mathbf{5 a}$ & $191-193$ & $\mathbf{6 a}$ & $160-162$ \\
\hline $\mathbf{3 b}$ & $151-153$ & - & - & $\mathbf{5 b}$ & $211-213$ & $\mathbf{6 b}$ & $219-221$ \\
\hline $\mathbf{3 c}$ & $168-169$ & $\mathbf{4 c}$ & $156-158$ & $\mathbf{5 c}$ & $178-180[27]$ & - & - \\
\hline $\mathbf{3 d}$ & $182-184$ & - & - & $\mathbf{5 d}$ & $222-223$ & $\mathbf{6 d}$ & $200-202$ \\
\hline $\mathbf{3 e}$ & $179-181$ & $\mathbf{4 e}$ & $173-174$ & $\mathbf{5 e}$ & $259-260[27]$ & - & - \\
\hline $\mathbf{3 f}$ & $143-145$ & $\mathbf{4 f}$ & $163-165$ & $\mathbf{5 f}$ & $244-246$ & - & - \\
\hline $\mathbf{3 g}$ & - & - & - & $\mathbf{5 g}$ & $225-227[27]$ & - & - \\
\hline
\end{tabular}

Table 2. The influence of reaction conditions on the yields of reaction products

\begin{tabular}{|c|c|c|c|}
\hline \multirow[t]{2}{*}{$\begin{array}{l}\text { Compd. } \\
\text { Index }\end{array}$} & \multirow[t]{2}{*}{ Reaction conditions } & \multicolumn{2}{|c|}{$\begin{array}{c}\text { Reaction products } \\
\text { yields }(\%)^{*}\end{array}$} \\
\hline & & 3 & $\sum \mathbf{4 , 5 , 6}$ \\
\hline $\mathbf{a}$ & \multirow{5}{*}{$\begin{array}{l}\mathrm{NEt}_{3} \text { in acetonitrile, } \\
3 \mathrm{~h} \text {, reflux temperature }\end{array}$} & 8 & 80 \\
\hline b & & 6 & 85 \\
\hline c & & 11 & 74 \\
\hline d & & 8 & 81 \\
\hline $\mathbf{e}$ & & 7 & 89 \\
\hline $\mathbf{a}$ & \multirow{5}{*}{$\begin{array}{c}\mathrm{NEt}_{3} \text { in acetonitrile, } \\
4 \text { days, } \mathrm{rt}\end{array}$} & 20 & 73 \\
\hline b & & 15 & 75 \\
\hline c & & 14 & 71 \\
\hline d & & 10 & 83 \\
\hline f & & 14 & 64 \\
\hline
\end{tabular}

In the reactions of benzimidazolium $N$-ylides generated from $N$-acetonyl-benzimidazolium chloride 1 with activated alkynes, the ratio of pyrrolo[1,2-a]benzimidazoles (3) to pyrrolo[1,2-a]quinoxaline derivatives (4-6) decreases by increasing the reaction temperature. We observed that the amount of pyrrolo[1,2-a]quinoxaline of type $\mathbf{4}$ or $\mathbf{6}$ decreases, while the amount of pyrrolo[1,2-a]quinoxalin-4-one 5 increases when the reaction temperature is increased. When ester-activated alkyne dipolarophiles are used, the ratio of pyrrolo[1,2-a]benzimidazoles 3 to pyrrolo[1,2- $a$ ]quinoxaline derivatives 4-6 is higher than in cases when ketone-activated alkyne dipolarophiles are used, as we reported earlier [27, 29]. In the reaction of 1-benzyl-3-acetonylbenzimidazolium chloride with 3-butyn-2-one in acetonitrile, in the presence of triethylamine, only pyrrolo[1,2-a]quinoxalin-4-one 5g was obtained (92\% yield at reflux temperature and $88 \%$ yield at room temperature) among traces of pyrrolo[1,2-a]benzimidazole $3 \mathrm{~g}$.

Only pyrrolo[1,2- $a$ ] benzimidazoles $\mathbf{3}$ and pyrrolo[1,2- $a$ ] quinoxalin-4-ones $\mathbf{5}$ are obtained when these reactions were carried out in the presence of oxidants, such as tetrapyridinecobalt(II)dichromate (TPCD) or $\mathrm{CrO}_{3}$ in $\mathrm{DMF}$ at $90^{\circ} \mathrm{C}$ (Table 3). The ratio between these two compounds is determined by the activated alkyne structure. Thus, starting from benzimidazolium $\mathrm{N}$-ylides generated from $\mathrm{N}$-acetonyl- 
benzimidazolium chloride 1 with ester-activated alkyne dipolarophiles, such as ethyl propiolate or dimethyl acetylenedicarboxylate (DMAD), in the presence of triethylamine and tetrapyridinecobalt(II)dichromate (TPCD), the major reaction products are pyrrolo[1,2-a]benzimidazoles 3 (50-70\%) and the minor reaction products are pyrrolo[1,2-a]quinoxalin-4-ones 5 (20-40\%). Using a ketoneactivated alkyne, such as 1-phenylpropynone, under the same reaction conditions, the major reaction products are pyrrolo[1,2-a]quinoxalin-4-ones $\mathbf{5}$ (70-80\%) and the minor reaction products are pyrrolo[1,2-a]benzimidazoles 3 (20-30\%). Easily separable pyrrolo[1,2-a]benzimidazoles $\mathbf{3}$ and pyrrolo[1,2-a]quinoxalin-4-ones 5 could be obtained in moderate to good yields in this way. Tuning the course of synthesis towards either pyrrolo[1,2-a]benzimidazole $\mathbf{3}$ or pyrrolo[1,2-a]quinoxalin-4-one $\mathbf{5}$ could be achieved by using the appropriate dipolarophile.

Table 3. The influence of the tpcd on the yields of reaction products

\begin{tabular}{|c|c|c|c|}
\hline \multirow[t]{2}{*}{$\begin{array}{l}\text { Compd. } \\
\text { index }\end{array}$} & \multirow[t]{2}{*}{ Reaction conditions } & \multicolumn{2}{|c|}{$\begin{array}{l}\text { Reaction products } \\
\text { yields }(\%)^{*}\end{array}$} \\
\hline & & 3 & $\sum 5,6$ \\
\hline $\mathbf{a}$ & \multirow{6}{*}{$\begin{array}{c}\mathrm{NEt}_{3}+\mathrm{TPCD} \\
\text { in } \mathrm{DMF}, \\
2 \mathrm{~h}, 90^{\circ} \mathrm{C}\end{array}$} & 51 & 48 \\
\hline b & & 21 & 77 \\
\hline c & & 55 & 44 \\
\hline d & & 26 & 68 \\
\hline $\mathbf{e}$ & & 68 & 26 \\
\hline f & & 62 & 26 \\
\hline
\end{tabular}

In order to explain the experimental data we proposed a reaction mechanism (Scheme 2) involving the initial formation of cycloadducts $\mathbf{A}$ which further undergo either an aromatization leading to pyrrolo[1,2-a]benzimidazole derivatives $\mathbf{3}$ or an imidazole ring opening giving pyrrole intermediates $\mathbf{B}$ depending on their stability. The presence of an ester group in position 3 of the primary cycloadducts $\mathbf{A}$ contributes to better stabilization of the intermediate structure $\mathbf{A}$ and to increased amounts of pyrrolo[1,2-a]benzimidazoles in comparison with compounds having a keto group in the same position 3 [29]. The formation of the pyrrole intermediates $\mathbf{B}$ involves the imidazole ring opening assisted by the abstraction of the acidic hydrogen from the $\mathrm{C}-1$ position of the primary cycloadduct $\mathbf{A}$. The nucleophilic addition of the secondary amino group to the keto group attached to the pyrrole ring in $\mathbf{B}$ gives cyclic carbinolamines with a pyrrolo[1,2-a]quinoxaline skeleton $\mathbf{C}$. Under the reaction conditions the carbinolamines $\mathbf{C}$ eliminate a molecule of water to form 4-methylene-pyrrolo[1,2- $a$ ] quinoxaline derivatives 4 . The separation and characterization of the open intermediates $\mathbf{B}$ has been reported as proof for the pathway to formation of pyrrolo[1,2-a]quinoxaline derivatives [28, 34].

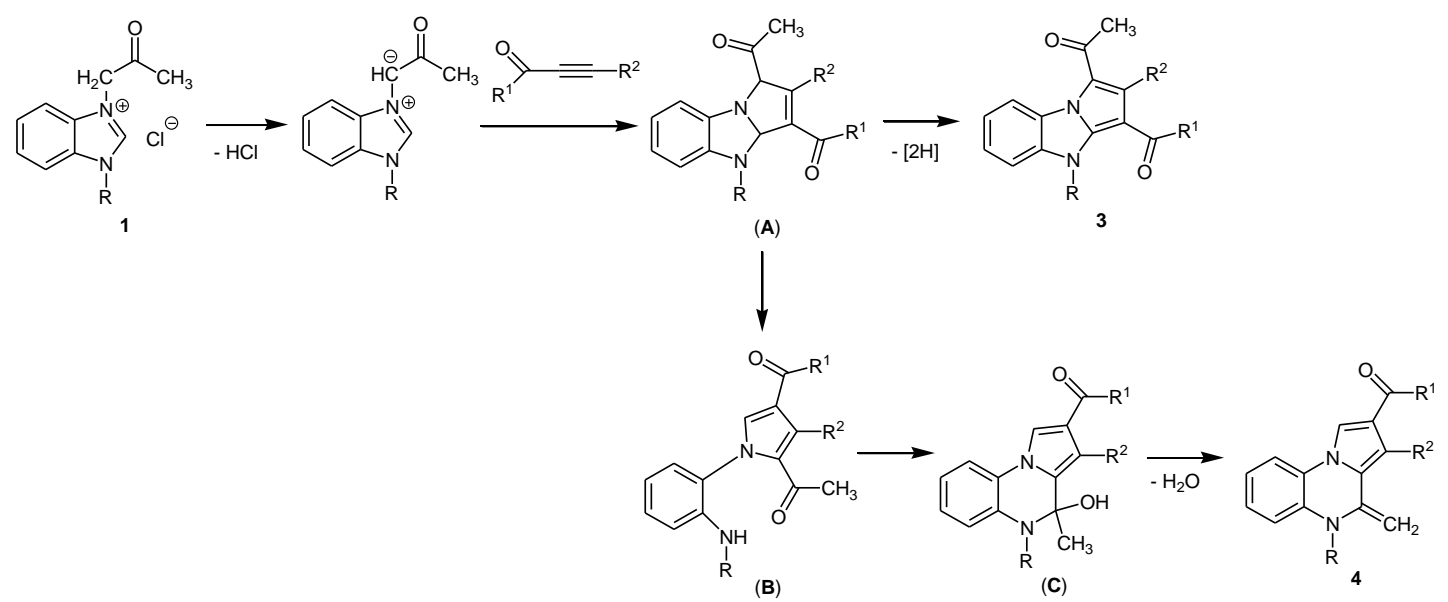

Scheme 2. The reaction mechanism for the formation of 4-methylene-pyrrolo[1,2-a]quinoxalines 4 
Due to their high reactivity, enamine compounds 4 could be transformed into pyrrolo[1,2a]quinoxalin-4-ones 5 in aqueous medium. In order to prove this type of transformation, we followed the conversion of $\mathbf{4 c}$ into $\mathbf{5 c}$ in $\mathrm{CDCl}_{3}$ solution. The experiment was performed in the NMR tube revealing the slow transformation of $\mathbf{4 c}$ into $\mathbf{5 c}$ in the $\mathrm{CDCl}_{3}$ solution under the influence of existing traces of water. This transformation was completed in about two weeks (Figure 3).

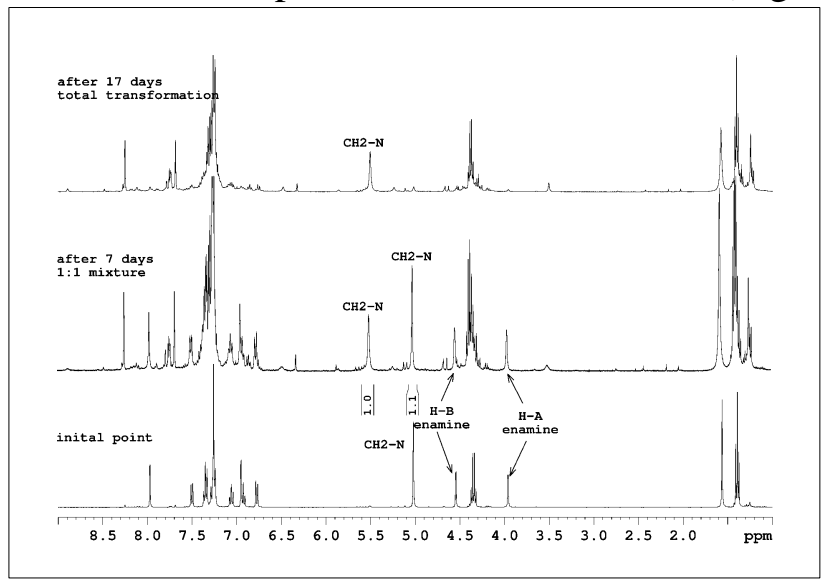

Figure 3. ${ }^{1} \mathrm{H}$ NMR spectrum of derivative $4 \mathbf{c}$ dissolved in $\mathrm{CDCl}_{3}$, recorded at different time intervals until its complete transformation into derivative $\mathbf{5 c}$ was achieved

The multinuclear NMR data recorded after 17 days for the resulting pyrrolo[1,2-a]quinoxalin-4-one 5c fully matched our previously reported data for this compound which we had isolated as a product of a different cycloaddition reaction [27].

We are reporting full, unambiguous assignments for all ${ }^{1} \mathrm{H},{ }^{13} \mathrm{C}$, and ${ }^{15} \mathrm{~N} N \mathrm{NM}$ chemical shifts of all described compounds, including the parent skeleton pyrrolo[1,2-a]quinoxaline enamines 4 and $\mathbf{6}$ (see Experimental section). The assignments are based on additional extensive 1D and 2D NMR experiments. Figure 4 shows a detail of the H,H-ROESY experiment which proves the through-space NOE interaction between $\mathrm{H}^{\mathrm{A}}$ in the terminal $=\mathrm{CH}_{2}$ enamine $(3.96 \mathrm{ppm})$ and $\mathrm{CH}_{2}-\mathrm{N}(5.02 \mathrm{ppm})$ groups in 4c. Another NOE interaction supporting the stereochemistry is that between $\mathrm{H}^{\mathrm{B}}$ in the terminal $=\mathrm{CH}_{2}$ enamine (4.64 $\mathrm{ppm})$ and the $\mathrm{H}-3$ proton $(7.00 \mathrm{ppm})$ from the five membered ring. The NMR assignments provided here should facilitate future identification of stereochemical isomers of pyrrolo[1,2- $a$ ]quinoxaline enamines 4 (i.e. $\mathrm{H}^{\mathrm{A}}$ cis to $\mathrm{N}-5$ and $\mathrm{H}^{\mathrm{B}}$ trans to $\mathrm{N}-5$ ). Similar ROESY experiments allowed us to assign the stereochemistry of conjugated double bonds in compounds 6.

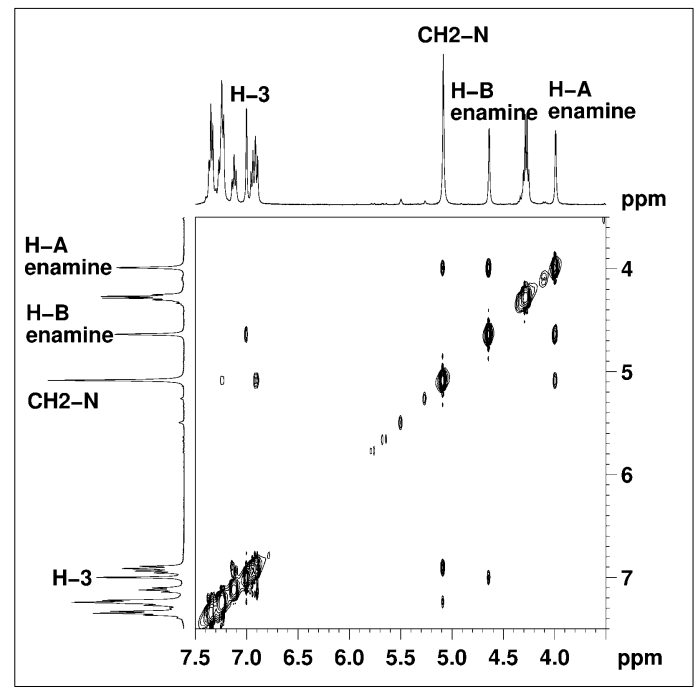

Figure 4. Detail of the H,H-ROESY experiment for $\mathbf{4 c}$ in

DMSO-d6 indicating the $\mathrm{H}^{\mathrm{A}} / \mathrm{H}^{\mathrm{B}}$ throughspace correlation in the $=\mathrm{CH}_{2}$ group (3.96/4.55 ppm), $\mathrm{H}^{\mathrm{A}} / \mathrm{CH}_{2}-\mathrm{N}$ (3.96/5.02 ppm) through-space correlation, as well as $\mathrm{H}^{\mathrm{B}} / \mathrm{H}-3$ (4.64/7.00 $\mathrm{ppm}$ ) through-space correlation 
The enamine structure of dimethyl 4-methylene-5-benzyl-4,5-dihydropyrrolo[1,2-a]quinoxalin-2,3dicarboxylate (4e) was also clearly confirmed for the first time by single crystal X-ray analysis (Figure $5)$.

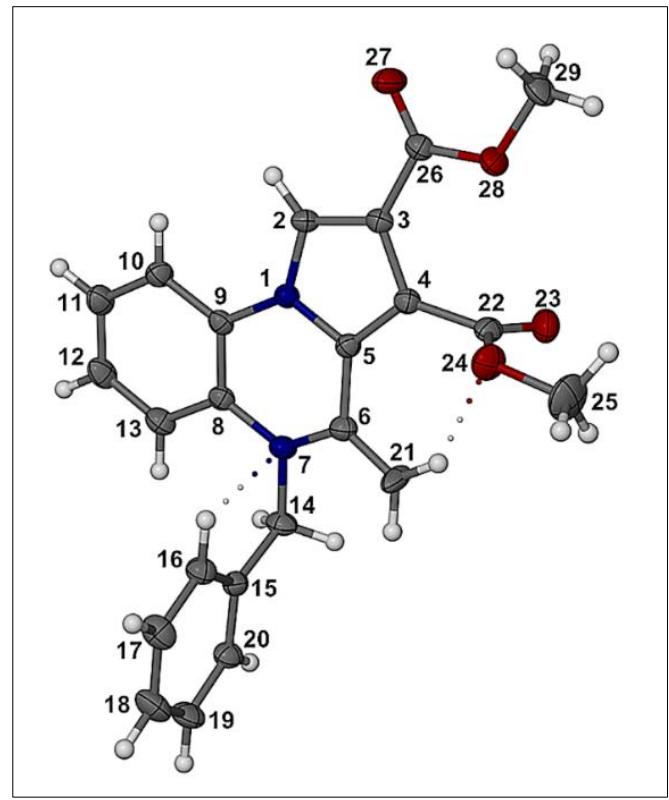

Figure 5. Molecular structure of $\mathbf{4 e}$ with crystallographic numbering and atoms drawn as thermal ellipsoids at the $50 \%$ probability level.

Intramolecular hydrogen bonds $(\mathrm{C}-\mathrm{H} \cdots \mathrm{N}, \mathrm{C}-\mathrm{H} \cdots \mathrm{O})$ that stabilize the observed conformation are shown as dotted lines

\section{Conclusions}

The formation of a mixture of pyrrolo[1,2-a]benzimidazoles 3, 4-methylene-pyrrolo[1,2a]quinoxalines 4 and pyrrolo[1,2-a]quinoxalin-4-ones 5 in 1,3-dipolar cycloaddition reactions of $N$ benzimidazolium ylides, generated in situ from $N$-acetonyl-benzimidazolium chlorides, with activated alkyne dipolarophiles was substantiated. We presented for the first time complete NMR and X-ray data for exocyclic enamine compounds of type 4. A mechanism explaining the formation of 4-methylenepyrrolo[1,2-a]quinoxalines $\mathbf{4}$ among pyrrolo[1,2- $a$ ] benzimidazoles $\mathbf{3}$ was also presented. Pyrrolo[1,2a]benzimidazoles 3 and pyrrolo[1,2-a] quinoxalin-4-ones 5 could be obtained in moderate to good yields by the direct 1,3-dipolar cycloaddition reactions of $N$-acetonyl-benzimidazolium chlorides with activated alkyne dipolarophiles in the presence of a strong base and oxidizing agents. The ratio between these two compounds is determined by the dipolarophile structures.

Acknowledgments: This work was supported by a grant of the Romanian National Authority for Scientific Research, CNCS-UEFISCDI, project number PN-III-P4-ID-PCCF-2016-0050 (5DnanoP). M.R.C. thanks the University of Cape Town and the National Research Foundation (Pretoria) for research support.-Access to research infrastructure developed in the "Petru Poni" Institute of Macromolecular Chemistry through the European Social Fund for Regional Development, Competitiveness Operational Programme Axis 1, Project InoMatPol (ID P_36_570, Contract 142/10.10.2016, cod MySMIS: 107464) is gratefully acknowledged.

\section{References}

1. ISLAM I., SKIBO E. B., DORR R. T., ALNERTS D. S., J. Med. Chem. 34, 1991, p. 2954.

2. SCHULZ W. G., NIEMAN R. A., SKIBO E. B., Proc. Nat. Acad. Sci. USA. 92, 1995, p. 11854.

3. CRAIGO W. A., LESUEUR B. W., SKIBO E. B., J. Med. Chem. 42, 1999, p. 3324.

4. HUANG X., SULEMAN A., SKIBO E. B., Bioorg. Chem. 28, 2000, p. 324.

5. HO W., MARYANOFF B. E., MCCOMSEY D. F., NORTEY S.O., U.S. Patent 5521200, 1996. Chem. Abstr. 125, 1996, 114628r. 
6. GUILLON J., LE BORGNE M., RIMBAULT C., MOREAU S., SAVRIMOUTOU S., PINAUD N., BARATIN S., MARCHIVIE M., ROCHE S., BOLLACKE A., PECCI A., ALVAREZ L., DESPLAT V., JOSE J., Eur. J. Med. Chem. 65, 2013, p. 205.

7. GUILLON J., REYNOLDS R. C., LEGER J.-M., GUIE M. A., MASSIP S., DALLEMAGNE P., JARRY C., J. Enz. Inh. Med. Chem. 19, 2004, p. 489.

8. GUILLON J., FORFAR I., MAMANI-MATSUDA M., DESPLAT V., SALIeGE M., THIOLAT D., MASSIP S., TABOURIER A., LÉGER J.-M., DUFAURE B., HAUMONT G., JARRY C., MOSSALAYI D., Bioorg. Med. Chem. 15, 2007, p. 194.

9. AGER IR, BARNES AC, DANSWAN GW, HAIRSINE PW, KAY DP, KENNEWELL PD, MATHARU SS, MILLER P, ROBSON P., J. Med. Chem. 31, 1988, p. 1098.

10. NEAMATI N., GAROFALO A., U.S. Patent 7947682, 2011.

11. GUILLON J., BOULOUARD M., LISOWSKI V., STIEBING S., LELONG V., DALLEMAGNE P., RAULT S., J. Pharm. Pharmacol. 52, 2000, p. 1369.

12. CAMPIANI G., MORELLI E., GEMMA S., NACCI V., BUTINI S., HAMON M., NOVELLINO E., GRECO G., CAGNOTTO A., GOEGAN M., CERVO L., DALLA VALLE F., FRACASSO C., CACCIA S., MENNINI T., J. Med. Chem. 42, 1999, p. 4362.

13. DAWOOD K. M., ABDEL-WAHABB B. F., ARKIVOC 2010, 333.

14. KALININ A. A., MAMEDOV V. A., Chem. Heterocycl. Compd. 46, 2011, p. 1423.

15. MAMEDOV V. A., KALININ A. A., Chem. Heterocycl. Compd. 46, 2010, p. 641.

16. OGURA H., KIKUCHI K., J. Org. Chem. 37, 1972, p. 2679.

17. ZUGRAVESCU I., HERDAN J., DRUTA I., Rev. Roum. Chim. 19, 1974, p. 649.

18. MATSUDA Y., YAMASHITA M., TAKAHASHI H., IDE S., TORISU K., FURUNO K., Heterocycles 33, 1992, p. 295.

19. WANG B., HU J., ZHANG X., HU Y., HU H., J Heterocycl Chem. 37, 2000, p. 1533.

20. METH-COHN O., Tetrahedron Lett. 6, 1975, p. 413.

21. ZHANG X., HUANG W., Tetrahedron Lett. 38, 1997, p. 4827.

22. ZHANG X., HUANG W., Tetrahedron. 54, 1998, p. 12465.

23. ZHANG Z., LI J., ZHANG G., MA N., LIU Q., LIU T., J. Org. Chem. 80, 2015, p. 6875.

24. PREETAM A., NATH M., RSC Advances 5, 2015, p. 21843.

25. NELINA-NEMTSEVA J. I., GULEVSKAYA A. V., Tetrahedron 72, 2016, p. 2327.

26. NICOLESCU A., DELEANU C., GEORGESCU E., GEORGESCU F., IURASCU A.-M., SHOVA S., FILIP P., Tetrahedron Lett. 54, 2013, p. 1486.

27. GEORGESCU E., NICOLESCU A., GEORGESCU F., TEODORESCU F., MARINESCU D., MACSIM A.-M., DELEANU C., Beilstein J. Org. Chem. 10, 2014, p. 2377.

28. GEORGESCU E., NICOLESCU A., GEORGESCU F., SHOVA S., TEODORESCU F., MACSIM A.-M., DELEANU C., Synthesis 47, 2015, p. 1643.

29. GEORGESCU E., NICOLESCU A., GEORGESCU F., TEODORESCU F., SHOVA S., MARINOIU A. T., DUMITRASCU F., DELEANU C., Tetrahedron 72, 2016, p. 2507.

30. GEORGESCU E., NICOLESCU A., GEORGESCU F., SHOVA S., SIMIONESCU B. C., DELEANU C., Rev. Roum. Chim. 61, 2016, p. 283.

31. SIMOVA S., Magn. Reson. Chem. 36, 1998, p. 505.

32. NICOLESCU A., BALAN M., GEORGESCU E., GEORGESCU F., URSU L., SIMIONESCU B.

C., FILIP P., DELEANU C., Rev Chim. (Bucharest) 64, 2013, p. 451.

33. WEY X., HU Z., LI T., HU H., J. Chem. Soc. Perkin. Trans. 1 1993, p. 2487.

34. MOLDOVEANU C., ZBANCIOC G., MANTU D., MAFTEI D., MANGALAGIU I., PLoS ONE 11, 2016, e0156129. doi:10.1371/journal.pone.0156129.

$\overline{\text { Manuscript received: 9.07.2019 }}$ 
